\title{
Commutativity conditions for truncated Brown-Peterson spectra of height 2
}

\author{
Tyler Lawson* Niko Naumann
}

October 26, 2018

\begin{abstract}
An algebraic criterion, in terms of closure under power operations, is determined for the existence and uniqueness of generalized truncated Brown-Peterson spectra of height 2 as $E_{\infty}$-ring spectra. The criterion is checked for an example at the prime 2 derived from the universal elliptic curve equipped with a level $\Gamma_{1}(3)$ structure.

2000MSC: 55P42, 55P43, 55N22 and 14L05
\end{abstract}

\section{Contents}

1 Introduction 2

2 Power operations 8

2.1 Total power operations ................ 8

2.2 Even-periodic objects . . . . . . . . . . . . . . . . . . 9 9

2.3 Homogeneous objects . . . . . . . . . . . . . . . 10

2.4 Height 1 objects . . . . . . . . . . . . . . . 11

*Partially supported by NSF grant 0805833 and a fellowship from the Sloan foundation. 
3 Realization problems for generalized $\mathrm{BP}\langle n\rangle$

12

$4 \quad K(2)$-local realization 18

$5 \quad K(1)$-local obstruction theory 22

5.1 The $K(n)$-local Adams-Novikov spectral sequence . . . . . . 2 22

$5.2 K(1)$-local complex oriented theories $\ldots \ldots \ldots . \ldots 24$

$5.3 K(1)$-local theories and periodicity $\ldots \ldots \ldots 28$

5.4 Obstruction theory . . . . . . . . . . . . . . 32

6 (1)-local realization 40

$6.1 \quad K(1)$-localized algebras . . . . . . . . . . . . . . 40

6.2 Application of obstruction theory . . . . . . . . . . . 41

$7 \quad K(0)$-local realization

8 An algebraic description of $\theta$ and the proof of Theorem 1.145

8.1 Computing $\theta \ldots \ldots \ldots \ldots \ldots$

8.2 Proof of Theorem $1.1 \ldots \ldots \ldots$

\section{Introduction}

The truncated Brown-Peterson spectra $\mathrm{BP}\langle n\rangle$ associated to a prime $p$ and an integer $n$, introduced in [JW73, are connective versions of the Landweber exact spectra $E(n)$ developed by Johnson and Wilson. The coefficient ring $\pi_{*} \mathrm{BP}\langle n\rangle=\mathbb{Z}_{(p)}\left[v_{1}, \ldots, v_{n}\right]$ parameterizes a simple family of formal group laws concentrated at chromatic heights 0 through $n$ and at height $\infty$.

These spectra $\mathrm{BP}\langle n\rangle$ have enjoyed recent prominence for the role they play in algebraic $K$-theory. Ausoni and Rognes [AR02, Introduction (0.2)] expect 
the existence of algebraic $K$-theory localization sequences,

$$
K\left(\mathrm{BP}\langle n-1\rangle_{p}^{\wedge}\right) \rightarrow K\left(\mathrm{BP}\langle n\rangle_{p}^{\wedge}\right) \rightarrow K\left(E(n)_{p}^{\wedge}\right),
$$

as part of a program to understand algebraic $K$-theory by applying it to the chromatic tower. For this and related applications it is useful to know whether these spectra admit highly associative $\left(A_{\infty^{-}}\right)$or highly commutative $\left(E_{\infty^{-}}\right)$ring structures. They are all known to admit $A_{\infty}$-ring structures [Laz03, discussion following Remark 2.12].

Only the simplest examples are known to admit $E_{\infty}$-ring structures: the spectra $\mathrm{BP}\langle-1\rangle=\mathbb{H} \mathbb{Z} / p, \mathrm{BP}\langle 0\rangle=\mathbb{H}_{(p)}$, and the spectra $\mathrm{BP}\langle 1\rangle$ [MS93, which are the Adams summands of connective $K$-theory. We remark that in exactly these cases, the existence of a localization sequence (1.1) is known. For $n=0$ this is classical, due to Quillen, and for $n=1$ it is work of Blumberg and Mandell [BM08, Introduction].

In this paper we construct a generalized $\mathrm{BP}\langle 2\rangle$ as an $E_{\infty}$-ring spectrum at the prime 2 , as follows.

Theorem 1.1. There exists a 2-local complex oriented $E_{\infty}$-ring spectrum $\operatorname{tmf}_{1}(3)_{(2)}$ such that the composite map of graded rings

$$
\mathbb{Z}_{(2)}\left[v_{1}, v_{2}\right] \subseteq B P_{*} \rightarrow\left(M U_{(2)}\right)_{*} \rightarrow \pi_{*}\left(\operatorname{tmf}_{1}(3)_{(2)}\right)
$$

is an isomorphism. Here the $v_{i}$ denote the 2-primary Hazewinkel generators. Any other such spectrum with an isomorphic formal group law is equivalent to $\operatorname{tmf}_{1}(3)_{(2)}$ as an $E_{\infty}$-ring spectrum.

In Section 3 we will discuss realization problems, and in particular how the existence of the above isomorphism is intrinsic to the formal group.

In forthcoming work [LN], the authors will show that there is a commutative diagram of $E_{\infty}$-ring spectra

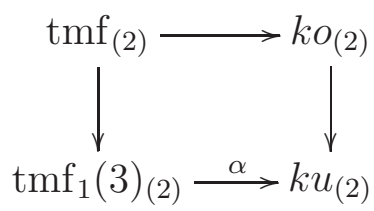


which, in mod 2 cohomology, induces the following canonical diagram of modules over the mod 2 Steenrod algebra $\mathcal{A}$ :

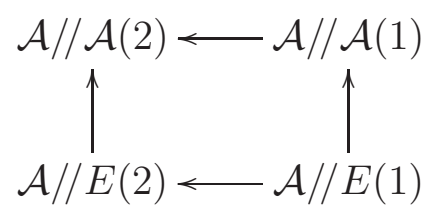

On homotopy groups, $\alpha$ induces the map sending $v_{1}$ to $v_{1}$ and $v_{2}$ to zero, and there is a consequent cofiber sequence of $\operatorname{tmf}_{1}(3)_{(2)}$-modules

$$
\Sigma^{6} \operatorname{tmf}_{1}(3)_{(2)} \stackrel{\cdot v_{2}}{\longrightarrow} \operatorname{tmf}_{1}(3)_{(2)} \stackrel{\alpha}{\longrightarrow} k u_{(2)} .
$$

The authors are grateful to Rognes for pointing out that this result might be useful in on-going joint work of Bruner and Rognes aimed at computing the algebraic $K$-groups $\pi_{*} K\left(\operatorname{tmf}_{(2)}\right)$.

In general, the possibility of constructing a truncated Brown-Peterson spectrum $\mathrm{BP}\langle n\rangle$ as an $E_{\infty}$-ring spectrum can be determined by iterative application of obstruction theory through a chromatic fracture cube. The necessary methods to carry this out are already well-understood, and were significantly applied in the construction of the spectrum of topological modular forms by Goerss, Hopkins, and Miller [GH, Beh]. These methods, when applied to $\mathrm{BP}\langle 2\rangle$, break down into the following stages:

- The Goerss-Hopkins-Miller theorem produces the $K(2)$-localized $E_{\infty^{-}}$ ring spectrum $L_{K(2)} \mathrm{BP}\langle 2\rangle$ as the homotopy fixed point object of a finite group action on a Lubin-Tate spectrum.

- The homotopy groups of the $K(1)$-localization $L_{K(1)} L_{K(2)} \mathrm{BP}\langle 2\rangle$ are equipped with a power operation $\theta$. These homotopy groups contain a subring consisting of the homotopy groups of $L_{K(1)} \mathrm{BP}\langle 2\rangle$. If this is a $\theta$-invariant subring, one produces an $E_{\infty}$-ring spectrum $L_{K(1)} \mathrm{BP}\langle 2\rangle$ equipped with a chromatic attaching map by use of Goerss-Hopkins obstruction theory [GH]. (If this is not a $\theta$-invariant subring, then $\mathrm{BP}\langle 2\rangle$ does not admit an $E_{\infty}$-ring structure.)

- The $E_{\infty}$-ring spectrum $L_{K(1) \vee K(2)} \mathrm{BP}\langle 2\rangle$ is then constructed by a chromatic pullback square. 
- The homotopy groups of $L_{K(0)} L_{K(1) \vee K(2)} \mathrm{BP}\langle 2\rangle$ contain as a subring the homotopy groups of $L_{K(0)} \mathrm{BP}\langle 2\rangle$. The latter is a free algebra on two generators, and this allows $L_{K(0)} \mathrm{BP}\langle 2\rangle$ to be realized as an $E_{\infty^{-}}$ ring spectrum, along with an arithmetic attaching map, by methods of rational homotopy theory.

- The object $L_{K(0) \vee K(1) \vee K(2)} \mathrm{BP}\langle 2\rangle$ is then constructed by a chromatic pullback square.

- $\mathrm{BP}\langle 2\rangle$ is the connective cover of $L_{K(0) \vee K(1) \vee K(2)} \mathrm{BP}\langle 2\rangle$, and canonically inherits an $E_{\infty}$-ring structure from $L_{K(0) \vee K(1) \vee K(2)} \mathrm{BP}\langle 2\rangle$ by [EKMM97, VII, 4.3].

More generally, these steps can be followed without starting with $\mathrm{BP}\langle 2\rangle$. Instead, one starts with a graded ring carrying a formal group law with similar chromatic data (but possibly determined as a quotient by a different regular sequence in $M U_{*}$ ). We will formalize this with the notion of a generalized $\mathrm{BP}\langle n\rangle$ - realization problem in Definition 3.1, If this realization problem can be solved, the solution is a generalized truncated Brown-Peterson spectrum as considered previously, such as in Str99b, Bak00.

The main result of this paper is Theorem 7.1. It shows that a formal group law over the graded ring $\mathbb{Z}_{(p)}\left[v_{1}, v_{2}\right]$, chromatically similar to that for $\mathrm{BP}\langle 2\rangle$, can be realized by an $E_{\infty}$-ring spectrum $R$ (which is essentially unique) if and only if the ring $\mathbb{Z}\left[\left(v_{2} / v_{1}^{p+1}\right)\right]_{p}^{\wedge}$ is stable under an induced power operation $\theta$. Moreover, any solution is unique up to weak equivalence. (This ring is a subring of the homotopy of a corresponding $K(1)$-localized Lubin-Tate spectrum). Work initiated by Ando And95, AHS04 allows an algebro-geometric interpretation of $\theta$ in terms of the associated formal group laws and descent data for level structures. It is perhaps surprising that the only problematic part of this general realization program is purely algebraic.

The proof of Theorem 7.1 occupies the bulk of the paper. By work of Rezk Rezb, at the prime 2 the power operations on the ring of modular forms of level $\Gamma_{1}(3)$ turn out to provide precisely the necessary algebraic descent data for level structures, and Theorem 1.1 follows. There are currently few instances where explicit computations of power operations on Lubin-Tate spectra of chromatic height 2 are known, and this is one of the main obstructions to extending Theorem 1.1 to primes $p \neq 2$ or to other generalized 
$\mathrm{BP}\langle 2\rangle$.

We remark that methods involving modular curves cannot extend to construct generalized versions of $\mathrm{BP}\langle 2\rangle$ at arbitrary primes. There are only finitely many modular curves having an underlying coarse moduli space which is a curve of genus zero with exactly one supersingular point, as demanded by the graded ring $\mathrm{BP}\langle 2\rangle_{*}$ together with its formal group. For the prime $p=3$ a similar method based on (derived) compact Shimura curves is shown to work in [HL10, Theorem 4.2]. On the other hand, Rezk's explicit formula in Proposition 8.4 is strictly more data than necessary. The final result does not require elliptic curves at all, but only a height 2 formal group law $\mathbb{G}$ over $\mathbb{Z}_{p} \llbracket a \rrbracket$ and a small subring of $\mathbb{Z}((a))_{p}^{\wedge}$ invariant under a lift of Frobenius which is canonically attached to $\mathbb{G}$. Using crystalline Diedonné theory, this existence statement translates into one concerning a rank $2 F$-crystal over $\mathbb{Z}_{p} \llbracket a \rrbracket$ with specific rationality properties. Though rather explicit, this problem remains open at present for all primes $p \neq 2,3$.

The current work is connected with the multiplicative ring spectrum project of Goerss and Hopkins Goe04 which attempts to lift algebraic diagrams to diagrams of $E_{\infty}$-ring spectra. The strongest currently available results in this direction are due to Lurie [Goe10, Theorem 4.7], and roughly produce the required $E_{\infty}$-ring spectra from suitable $p$-divisible groups. These in turn can be produced from families of elliptic curves (leading to TMF [Lur09]) and, more generally, from suitable abelian varieties (leading to TAF [BL10]).

However, there are many more $p$-divisible groups than there are abelian varieties. This flexibility allowed specifically constructed $p$-divisible groups to realize the localizations $L_{K(2) \vee \cdots \vee K(n)} E(n)$ of generalized Johnson-Wilson spectra as $E_{\infty}$-ring spectra [Law10, Example 5.4] Given appropriate algebraic data, the spectrum $L_{K(1)} E(n)$ and its chromatic attaching map are directly realizable by the Goerss-Hopkins obstruction theory, and the question of whether an $E_{\infty}$-ring structure can be placed on the spectrum $E(n)$ is reduced to a very explicit, though potentially hard, arithmetic existence problem.

We conclude this section by giving an overview of the paper.

In Section 2 we recall fundamental results about power operations with an emphasis on the $K(1)$-local case. 
Section 3 sets up the realization problems for generalized $\mathrm{BP}\langle n\rangle$ and introduces some technical material: for understanding how complex orientations can be lifted along a map of ring spectra, and for rigidifying certain group actions.

Section 4 shows that, as a consequence of the Goerss-Hopkins-Miller theorem and realizability results of Baker and Richter, the realization problem for $\mathrm{BP}\langle 2\rangle$ always has an essentially unique solution $K(2)$-locally, given by a homotopy fixed point spectrum of a Lubin-Tate spectrum.

Section 5, studying $K(1)$-local obstruction theory, is the technical heart of the paper. It leads up to the proof of the main $K(1)$-local existence and uniqueness result, Theorem 5.20.

Section 6 deduces that closure under the power operation $\theta$ is necessary and sufficient to extend a $K(2)$-local solution to a given existence problem to a $K(1) \vee K(2)$-local solution, and that any such extension is unique up to equivalence.

Section 7 completes the proof characterizing existence and proving uniqueness for realizing a generalized $\mathrm{BP}\langle 2\rangle$, Theorem 7.1 , by methods of rational homotopy theory.

Section 8 provides a purely algebraic description of the power operation $\theta$ and uses elliptic curves with $\Gamma_{1}(3)$-level structures to complete the proof of Theorem 1.1.

Throughout this paper, we follow the standard conventions that, for a spectrum $E$, we write $\pi_{*} E=E_{*}=E^{-*}$.

\section{Acknowledgements}

The authors would like to thank Andrew Baker, Daniel Davis, and John Rognes for discussions of some of the present material, and the anonymous referees for a number of observant remarks and the suggestion to study uniqueness. 


\section{Power operations}

Let $p$ be a prime and $E$ an $E_{\infty}$-ring spectrum which is $p$-local and complex orientable. In this section we review the necessary details from AHS04. about power operations on E-cohomology. The authors have been heavily influenced by the work of Rezk [Reza].

\subsection{Total power operations}

For any element $\alpha \in E^{0}(X)=\left[\Sigma_{+}^{\infty} X, E\right]$, composing the $p$-fold symmetric power of this map with the multiplication map of $E$ gives rise to a power operation

$$
P(\alpha): \Sigma_{+}^{\infty}\left(B \Sigma_{p} \times X\right) \rightarrow E .
$$

The transformation $P: E^{0}(X) \rightarrow E^{0}\left(X \times B \Sigma_{p}\right)$ is natural in spaces $X$ and $E_{\infty}$-ring spectra $E$. It preserves multiplication but not addition (there is a Cartan formula), and composing $P(\alpha)$ with the inclusion $X \rightarrow B \Sigma_{p} \times X$ gives $\alpha^{p}$.

Let $I_{X}$ be the ideal which is the image of the transfer $\operatorname{Tr}: E^{0}(X) \rightarrow E^{0}(X \times$ $\left.B \Sigma_{p}\right)$; then $I_{X}$ is the principal ideal generated by $\operatorname{Tr}(1) \in E^{0}\left(B \Sigma_{p}\right)$. As $E$ is $p$-local, this ideal coincides with the image of the transfer from $E^{0}(X \times$ $\left.B\left(\Sigma_{r} \times \Sigma_{s}\right)\right)$ associated to any subgroup $\Sigma_{r} \times \Sigma_{s}$ for $0<r, s<p, r+s=p$. The resulting natural transformation

$$
\psi_{X}^{p}: E^{0}(X) \stackrel{P}{\longrightarrow} E^{0}\left(X \times B \Sigma_{p}\right) \longrightarrow E^{0}\left(X \times B \Sigma_{p}\right) / I_{X}
$$

is a ring homomorphism. We simply write $\psi^{p}=\psi_{X}^{p}$ when $X$ is a point.

The image of $\operatorname{Tr}(1)$ under the map $E^{0}\left(B \Sigma_{p}\right) \rightarrow E^{0}(*)$ is $p$ !, and hence we obtain a factorization as follows:

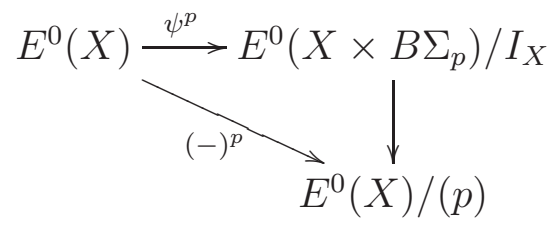

The ring homomorphism $\psi^{p}$ therefore reduces, modulo the ideal $(p)$, to the Frobenius ring homomorphism $x \mapsto x^{p}$ on $E^{0}(X) /(p)$. 
Let $j: C_{p} \hookrightarrow \Sigma_{p}$ be a monomorphism from a cyclic subgroup of order $p$. The index $\left[\Sigma_{p}: C_{p}\right]$ is prime to $p$, so the image of $I_{X}$ under the restriction map $E^{0}\left(X \times B \Sigma_{p}\right) \rightarrow E^{0}\left(X \times B C_{p}\right)$ coincides with the image of the transfer morphism $E^{0}(X) \rightarrow E^{0}\left(X \times B C_{p}\right)$.

Consider the case $X=B C_{p}$. Write $\epsilon: E^{0}\left(B C_{p}\right) \rightarrow E^{0}$ for the map induced by the homomorphism $\{e\} \rightarrow C_{p}$ from the trivial group. For any $\alpha \in E^{0}\left(B C_{p}\right)$, we have the following diagram, which commutes up to homotopy:

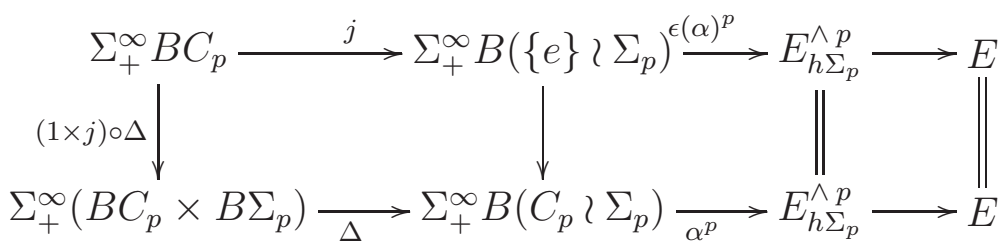

(Here the wreath product $G \succ \Sigma_{p}$ denotes the semidirect product $G^{p} \rtimes \Sigma_{p}$, with the symmetric group acting by permutation.) The left-hand square commutes up to homotopy because the two composite maps are induced by two conjugate group homomorphisms $C_{p} \rightarrow C_{p} 2 \Sigma_{p}$. As a consequence, we have the following commutative diagram of total power operations:

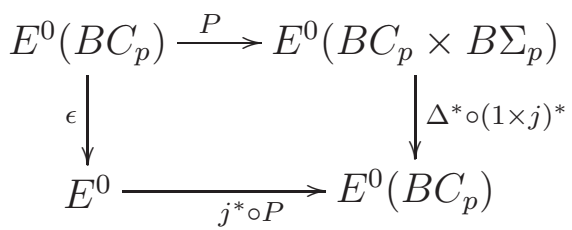

\section{$2.2 \quad$ Even-periodic objects}

In this section we assume that our fixed $p$-local, complex orientable $E_{\infty}$-ring spectrum $E$ is even-periodic, i.e. that for all integers $m, E^{2 m+1}$ is zero and there is a unit in $E^{2}$.

Under these circumstances, the function spectrum $E^{X}=F\left(\Sigma_{+}^{\infty} X, E\right)$ is an $E_{\infty}$-ring spectrum which inherits a complex orientation from $E$. The ring $E^{0}\left(X \times \mathbb{C P}^{\infty}\right)$ is the coordinate ring of a formal group $\mathbb{G}$ over $E^{0}(X)$ Str99a, Section 8]. Naturality of the map $\psi_{(-)}^{p}$ with respect to the coordinate projections and multiplication on $\mathbb{C P}^{\infty} \times \mathbb{C P}^{\infty}$ implies that the map

$$
\psi_{\mathbb{C P}^{\infty}}^{p}: E^{0}\left(\mathbb{C P}^{\infty}\right) \rightarrow E^{0}\left(\mathbb{C P}^{\infty} \times B \Sigma_{p}\right) / I_{\mathbb{C P}}
$$


is a homomorphism $\mathbb{G} \rightarrow\left(\psi^{p}\right)^{*} \mathbb{G}$ of formal groups which is a lift of the Frobenius isogeny. More is true. When the formal group has constant height, the ring $E^{0}\left(B \Sigma_{p}\right) / I$ is the universal $E^{0}$-algebra classifying subgroup schemes of $\mathbb{G}$ of rank $p$, and consideration of equation (2.1) implies that this universal subgroup scheme is the kernel of the isogeny $\psi_{\mathbb{C P}}^{p}$.

Let $C_{p} \subseteq S^{1}$ be the unique cyclic subgroup of order $p$. It induces a map $B C_{p} \rightarrow B S^{1} \cong \mathbb{C P}^{\infty}$. If $p$ is not a zero divisor in $E^{*}$, the map $E^{0}\left(\mathbb{C P}^{\infty}\right) \rightarrow$ $E^{0}\left(B C_{p}\right)$ is a quotient map, and $E^{0}\left(B C_{p}\right)$ is the coordinate ring of the $p$ torsion subgroup of the formal group.

\subsection{Homogeneous objects}

In this section we assume that $E$ is even-periodic, that the ring $E^{0}$ is a $p$ torsion-free complete local ring with maximal ideal $\mathfrak{m}$ containing $p$, and that the mod-m reduction of the formal group on $E^{0}$ is of constant height $n<\infty$.

Under these circumstances, the power operations on $E$-cohomology give rise to the following result. To formulate it, we will write $f^{*} \mathbb{G}$ for the image of a formal group $\mathbb{G}$ along a ring homomorphism $f$.

Theorem 2.1 ([AHS04, Theorem 3.25]). The ring $E^{0}$ with formal group $\mathbb{G}$ has descent data for level structures, as follows. The pair

$$
\left(E^{0}\left(B \Sigma_{p}\right) / I, \operatorname{Spec}\left(E^{0}\left(B C_{p}\right)\right) \subseteq \psi^{*} \mathbb{G}\right)
$$

is the universal example of a pair $(S, H)$ consisting of an $E^{0}$-algebra $f: E^{0} \rightarrow$ $S$ together with a subgroup $H \subseteq f^{*} \mathbb{G}$ of rank $p$. Given such a pair $(S, H)$, one obtains a classifying map of $E^{0}$-algebras $F: E^{0}\left(B \Sigma_{p}\right) / I \rightarrow S$. The resulting ring homomorphism $f^{(p)}=F \circ \psi: E^{0} \rightarrow S$ comes with an isogeny $\psi_{\mathbb{C P} \infty}^{p}: f^{*} \mathbb{G} \rightarrow\left(f^{(p)}\right)^{*} \mathbb{G}$ with kernel $H$. These fit into a commutative diagram as follows:

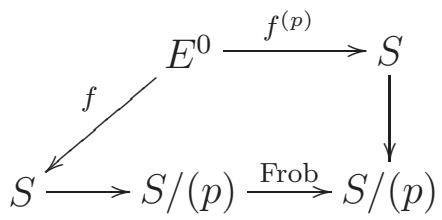

Remark 2.2. The reference AHS04, in order to guarantee the existence of universal deformations, makes the blanket assumption that $E^{0}$ is a complete 
local ring with perfect residue field of characteristic $p>0$. In the course of this paper we will have need for Theorem 2.1 in the case where the residue field is not perfect; we note that the proof of the above theorem is a formal consequence of calculations in E-cohomology and does not require a perfect residue field.

One particular case is when $E$ is the Lubin-Tate cohomology theory associated to a formal group law $\mathbb{G}_{0}$ of height $n<\infty$ over a perfect field $k$ of characteristic $p$ [Rez98, GH]. In this case, we have the following algebraic description of the descent structure coming from the $E_{\infty}$-structure.

Proposition 2.3 ([AHS04, Proposition 12.12]). If $E$ is a Lubin-Tate cohomology theory, the descent data for level structures on $E^{0}$ provided by the (unique) $E_{\infty}$-structure of Goerss and Hopkins coincides with the descent data provided by the universal property of the Lubin-Tate ring.

\section{$2.4 \quad$ Height 1 objects}

In this section we discuss the power operations of the previous section in the height 1 case. In this case, any formal group $\mathbb{G}$ of constant height 1 has a unique subgroup scheme of rank $p$ given by the kernel of multiplication-by- $p$, which we denote by $\mathbb{G}[p] \subseteq \mathbb{G}$. The ring $E^{0}\left(B \Sigma_{p}\right) / I$ classifying subgroups of rank $p$ is therefore isomorphic to $E^{0}$, and the power operation takes the form of a map $\psi^{p}: E^{0} \rightarrow E^{0}$ which is a lift of Frobenius.

Conversely, suppose we are given a $p$-adic ring $E$ with formal group $\mathbb{G}$ of constant height 1, together with a lift of Frobenius $\psi: E \rightarrow E$. Write $\overline{\mathbb{G}}$ for the mod- $p$ reduction of $\mathbb{G}$ and $\overline{\mathbb{G}}^{(p)}$ for the pullback of $\overline{\mathbb{G}}$ along the Frobenius map. In this case, rigidity of formal tori says that the canonical homomorphism

$$
\operatorname{Hom}_{E}\left(\mathbb{G}, \psi^{*} \mathbb{G}\right) \rightarrow \operatorname{Hom}_{E /(p)}\left(\overline{\mathbb{G}}, \overline{\mathbb{G}}^{(p)}\right),
$$

between abelian groups of homomorphisms of formal groups over the indicated base rings, is a bijection. This is easier to see on the dual groups $\mathbb{G}^{\vee}$ and $\left(\psi^{*} \mathbb{G}\right)^{\vee}$, which are étale, by using the fact that the reduction $E \rightarrow E /(p)$ induces an equivalence of étale sites. In particular, there is a unique lift of the relative Frobenius isogeny $\overline{\mathbb{G}} \rightarrow \overline{\mathbb{G}}^{(p)}$ over $E /(p)$ to an isogeny $\mathbb{G} \rightarrow \psi^{*} \mathbb{G}$ over $E$, necessarily of degree $p$. 
Now suppose $F$ is an $E_{\infty}$-ring spectrum satisfying the assumptions stated at the beginning of Section 2.3, and define $E=L_{K(1)} F$. The natural localization map $F \rightarrow E$ gives rise to a diagram of total power operations:

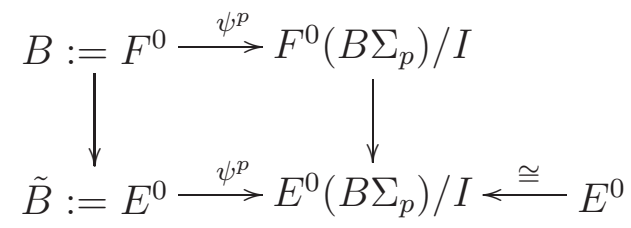

Naturality of the description of $\psi^{p}$ implies that the right-hand vertical map can be expressed in terms of descent data. Specifically, the formal group $\mathbb{G}_{B}$ over $B$ determined by the complex orientability of $F$ has a unique subgroup scheme $C$ of rank $p$ after being pulled back to $\tilde{B}=E^{0}$, and the composite map $F^{0}\left(B \Sigma_{p}\right) / I \rightarrow E^{0}\left(B \Sigma_{p}\right) / I \cong E^{0}$ classifies this subgroup. In other words, there is an isomorphism

$$
\left(\psi^{p}\right)^{*} \mathbb{G}_{\tilde{B}} \cong \mathbb{G}_{\tilde{B}} / C
$$

over $\tilde{B}=E^{0}$.

\section{Realization problems for generalized $\mathrm{BP}\langle n\rangle$}

In this section we describe a family of graded rings, together with formal group laws, that share chromatic features with the formal group law of $\mathrm{BP}\langle n\rangle$. Our aim is to understand when these can be realized as the homotopy of complex oriented $E_{\infty}$-ring spectra. The chromatic machinery described in this paper iteratively realizes various algebras over these rings, and this section also provides some technical tools for understanding the formal group data of a spectrum based on knowledge of the formal group data of an algebra over it.

Definition 3.1. Fix a prime $p$ and an integer $n \geq 0$. A (generalized) $\mathrm{BP}\langle n\rangle$ realization problem is a graded $\mathbb{Z}_{(p)}$-algebra $A$, equipped with a map $M U_{*} \rightarrow$ $A$ classifying a graded formal group law $\mathbb{G}$, such that

- $A$ is concentrated in nonnegative degrees, 
- $A$ is degreewise finitely generated free over $\mathbb{Z}_{(p)}$,

- the sequence $p, v_{1}, \ldots, v_{n}$ in $A$ determined by $\mathbb{G}$ is a regular sequence, and

- the quotient of $A$ by the ideal $\left(p, v_{1}, \ldots, v_{n}\right)$ is isomorphic to $\mathbb{F}_{p}$ concentrated in degree zero.

Remark 3.2. This definition admits several equivalent formulations. One of these is that the composite map

$$
\mathbb{Z}_{p}\left[v_{1}, \ldots, v_{n}\right] \subseteq B P_{*} \rightarrow\left(M U_{(p)}\right)_{*} \rightarrow A
$$

is an isomorphism. Another is that $A$ is a quotient of $B P_{*}$ by a regular sequence given by lifts of the classes $v_{k}$ for $k>n$. The given definition depends only on the formal group rather than the formal group law, and does not depend on choices of lifts of the classes $v_{m}$, which are only uniquely determined $\bmod \left(p, v_{1}, \ldots, v_{m-1}\right)$.

Definition 3.3. Suppose $(A, \mathbb{G})$ is a (generalized) $\mathrm{BP}\langle n\rangle$-realization problem. For any graded $A$-algebra $B$, a solution to this realization problem for $B$ is a complex oriented $E_{\infty}$-ring spectrum $T$, equipped with an isomorphism of graded rings $T_{*} \rightarrow B$ which sends the formal group of $T$ to the image of the formal group $\mathbb{G}$ over $B$.

Two solutions $T$ and $T^{\prime}$ are equivalent if there exists a weak equivalence $T \rightarrow T^{\prime}$ of $E_{\infty}$-ring spectra which, on homotopy groups, is compatible with the given identifications of $B$ with $T_{*}$ and $T_{*}^{\prime}$.

Definition 3.4. Suppose $(A, \mathbb{G})$ is a (generalized) $\mathrm{BP}\langle n\rangle$-realization problem, and recall the consequent isomorphism $A \cong \mathbb{Z}_{(p)}\left[v_{1}, \ldots, v_{n}\right]$. For any graded $A$-algebra $B$ and $0 \leq m \leq n$, we define the " $K(m)$-localization" of $B$ to be the $B$-algebra

$$
B_{K(m)}=\left(v_{m}^{-1} B\right)_{\left(p, v_{1}, \ldots, v_{m-1}\right)}^{\wedge} .
$$

When $m=0$ we follow the usual convention that $v_{0}=p$. Here the completion of a graded ring by a homogeneous ideal denotes the completion in the category of graded rings. 
Remark 3.5. The following are some cases relevant for the present paper. Suppose $A$ is the graded ring $\mathbb{Z}_{(p)}\left[v_{1}, v_{2}\right]$ with $\left|v_{i}\right|=2\left(p^{i}-1\right)$, and let $x=$ $v_{1}^{p+1} v_{2}^{-1}$, of degree 0 . In the following, $R\{\cdots\}$ denotes a free $R$-module with indicated generators.

$$
\begin{array}{rlrl}
A_{K(0)} & =\mathbb{Q}\left[v_{1}, v_{2}\right] & & \text { and }\left(A_{K(0)}\right)_{0}=\mathbb{Q} . \\
A_{K(1)} & =\mathbb{Z}_{p}\left[x^{-1}\right]_{p}^{\wedge}\left[v_{1}^{ \pm 1}\right] & & \text { and }\left(A_{K(1)}\right)_{0}=\mathbb{Z}_{p}\left[x^{-1}\right]_{p}^{\wedge} . \\
A_{K(2)} & =\mathbb{Z}_{p} \llbracket x \rrbracket\left[v_{2}^{ \pm 1}\right] \cdot\left\{1, v_{1}, \ldots, v_{1}^{p}\right\} & \\
& =\mathbb{Z}_{p} \llbracket x \rrbracket\left[v_{2}^{ \pm 1}, v_{1}\right] /\left(v_{1}^{p+1}-x v_{2}\right) & & \text { and }\left(A_{K(2)}\right)_{0}=\mathbb{Z}_{p} \llbracket x \rrbracket . \\
A_{K(2), K(1)} & =\left(\mathbb{Z}_{p}((x))_{p}^{\wedge}\right)\left[v_{1}^{ \pm 1}\right] &
\end{array}
$$

For the last, there are several possible ways to describe the degree-0 part:

$$
\begin{aligned}
\left(A_{K(2), K(1)}\right)_{0} & =\left(\mathbb{Z}_{p} \llbracket x \rrbracket\left[x^{-1}\right]\right)_{p}^{\wedge} \\
& =\mathbb{Z}_{p}((x))_{p}^{\wedge} \\
& =\left\{\sum_{n \in \mathbb{Z}} \alpha_{n} x^{n} \mid \alpha_{n} \in \mathbb{Z}_{p}, \alpha_{n} \rightarrow 0 \text { as } n \rightarrow-\infty\right\}
\end{aligned}
$$

Remark 3.6. The interest in this algebraic construction is as follows. If the generalized $\mathrm{BP}\langle n\rangle$-realization problem has a solution $R$, one can check that

$$
\pi_{*}\left(L_{K(n)} R\right) \cong\left(\pi_{*} R\right)_{K(n)}
$$

as graded algebras over $\pi_{*}(R) \cong A$. The generalizations to iterated application of $K(i)$-localization for $0 \leq i \leq n$ are also true. One may prove this result using [Hov95, Theorem 1.5.4] by first showing that $R$ satisfies the telescope conjecture using [Hov97, Theorem 1.9], or alternatively by using the results of [GM95], in a way generalizing the proof of [Beh, Lemma 8.1].

For the remainder of this paper we concentrate on generalized $\operatorname{BP}\langle 2\rangle$-realization problems, and simply refer to them as realization problems.

The following two lemmas will prove convenient for lifting formal group data to a homotopy commutative ring spectrum $R$ from a given $R$-algebra $S$.

Lemma 3.7. Suppose $f: A \rightarrow B$ is a faithfully flat map of commutative rings and $A$ is torsion-free. If $\mathbb{G}, \mathbb{G}^{\prime}$ are two formal group laws over $A$ such that $f^{*} \mathbb{G}$ and $f^{*} \mathbb{G}^{\prime}$ are strictly isomorphic over $B$, then $\mathbb{G}$ and $\mathbb{G}^{\prime}$ are strictly isomorphic over $A$. 
Proof. Let $h(x)=x+\sum b_{n} x^{n+1}$ be a strict isomorphism between $\mathbb{G}$ and $\mathbb{G}^{\prime}$ with coefficients $b_{n} \in B$. Consider the ring $B \otimes_{A} B$, equipped with two unit maps $\eta_{L}, \eta_{R}: B \rightarrow B \otimes_{A} B$. We define $g$ to be the common composite $\eta_{L} \circ f=$ $\eta_{R} \circ f$. The power series $\eta_{L}^{*}(h)$ and $\eta_{R}^{*}(h)$ provide two strict isomorphisms $g^{*} \mathbb{G} \rightrightarrows g^{*} \mathbb{G}^{\prime}$.

The map $g$ is flat. Hence $B \otimes_{A} B$ is torsion-free, and embeds into its rationalization $\left(B \otimes_{A} B\right) \otimes \mathbb{Q}$. Over this ring the composite $\eta_{L}^{*}(h)^{-1} \circ \eta_{R}^{*}(h)$ is a strict automorphism of $\left(g^{*} \mathbb{G}\right)_{\mathbb{Q}}$, which is isomorphic to the additive group. The only strict automorphism of the additive group in characteristic zero is the identity, and hence $\eta_{L}^{*}(h)=\eta_{R}^{*}(h)$.

The power series $h$ thus has coefficients in the equalizer of the maps

$$
\eta_{L}, \eta_{R}: B \rightrightarrows B \otimes_{A} B
$$

However, faithfully flat descent implies that this equalizer is precisely $A$.

Corollary 3.8. Suppose $f: R \rightarrow S$ is a map of complex oriented, homotopy commutative ring spectra such that $R_{*}$ and $S_{*}$ are concentrated in even degrees, $R_{*}$ is torsion-free, and the induced map $f_{*}: R_{*} \rightarrow S_{*}$ is faithfully flat. Suppose that, on homotopy groups, the orientation $g: M U \rightarrow S$ has a factorization $M U_{*} \rightarrow R_{*} \rightarrow S_{*}$. Then the orientation $g$ itself factors through $R$.

Proof. We have a second orientation $g^{\prime}: M U \rightarrow R \stackrel{f}{\rightarrow} S$, given by the composite of any orientation of $R$ with the map $f$. The two induced maps $g_{*}, g_{*}^{\prime}: M U_{*} \rightarrow S_{*}$ both factor through $R_{*}$ by assumption, and as they arise from two orientations $M U \rightarrow S$ the two formal group laws classified by $g_{*}$ and $g_{*}^{\prime}$ over $S_{*}$ are strictly isomorphic. Lemma 3.7 shows that there is a strict isomorphism of these formal group laws over $R_{*}$. The result follows: any choice of orientation $M U \rightarrow R$, with induced formal group law $\mathbb{G}$, establishes a bijection between homotopy classes of ring spectrum maps $M U \rightarrow R$ and strict isomorphisms with domain $\mathbb{G}$.

Lemma 3.9. Suppose that we have a homotopy pullback diagram

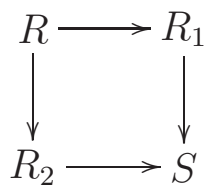


of homotopy commutative ring spectra such that $S$ is complex orientable and $S_{*}$ is concentrated in even degrees. Then the set of complex orientations of $R$ maps isomorphically to the pullback of the sets of complex orientations of $R_{1}$ and $R_{2}$ over the set of complex orientations of $S$.

Proof. Recall that a complex orientation of $R$ is the same as the homotopy class of a map of ring spectra $M U \rightarrow R$. By [HS99, Proposition 2.16] and the classical computation $M U_{*} M U \cong M U_{*}\left[b_{i}\right]$ with $\left|b_{i}\right|=2 i(i \geq 1)$, we have isomorphisms

$$
S_{*} M U \cong S_{*}\left[b_{i}\right] \text { and } S^{*} M U \cong \operatorname{Hom}_{S_{*}}\left(S_{*} M U, S_{*}\right)
$$

In particular, both groups are concentrated in even degrees by the assumption on $S_{*}$, and the Mayer-Vietoris sequence degenerates to a pullback diagram

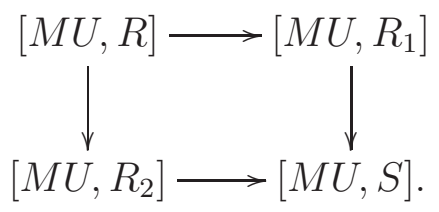

The same argument applies with $\mathbb{S}$ and $M U \wedge M U$ in place of $M U$. Therefore, restricting to the subsets of maps commuting with the unit map and multiplication map, we obtain a pullback diagram of sets of homotopy classes of ring spectrum maps, as desired.

The following lemma applies Cooke's obstruction theory [Coo78] to lift group actions and equivariant maps from the homotopy category of $E_{\infty}$-ring spectra to honest actions. Recall all spectra are implicitly assumed to be $p$-local for a fixed prime $p$. These techniques have been previously applied to the construction of group actions on $E_{\infty}$-ring spectra [Beh, Szy11.

Lemma 3.10. Let $G$ be a finite group of order prime to $p$.

1. Suppose that $R$ is an $E_{\infty}$-ring spectrum. Any action of $G$ on $R$ in the homotopy category of $E_{\infty}$-ring spectra has a unique lift, up to weak equivalence, to an action of $G$ on $R$ in the category of $E_{\infty}$-ring spectra. 
2. Let $R$ and $S$ be $E_{\infty}$-ring spectra, equipped with actions of $G$ in the category of $E_{\infty}$-ring spectra. Suppose that $\pi_{1} \operatorname{Map}_{E_{\infty}}(R, S)$ is abelian for all choices of basepoint. Then the natural map

$$
\pi_{0} \operatorname{Map}_{E_{\infty}}^{G}(R, S) \rightarrow\left(\pi_{0} \operatorname{Map}_{E_{\infty}}(R, S)\right)^{G},
$$

from the connected components of the derived mapping space of $G$ equivariant $E_{\infty}$-ring spectra maps to $G$-invariant components of the derived $E_{\infty}$-mapping space, is an isomorphism.

Proof. Let $f: R \rightarrow S$ be a map of $E_{\infty}$-ring spectra. Without loss of generality, assume that $R$ and $S$ are fibrant-cofibrant and that the map $f$ is a cofibration. We define $\operatorname{hAut}_{E_{\infty}}(R) \subseteq \operatorname{Map}_{E_{\infty}}(R, R)$ to be the topological submonoid consisting of homotopy equivalences; this is a union of path components. This allows us to introduce a monoid of homotopy automorphisms of the map $f$ by a pullback diagram of mapping spaces (which is a homotopy pullback) as follows:

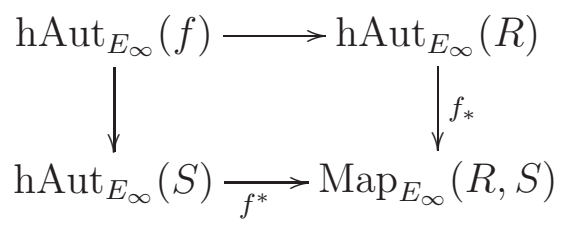

We will show that, for any map $f: R \rightarrow S$ of $E_{\infty}$-ring spectra and any homomorphism

$$
G \rightarrow \pi_{0} \mathrm{hAut}_{E_{\infty}}(R) \times_{\pi_{0} \operatorname{Map}_{E_{\infty}}(R, S)} \pi_{0} \mathrm{hAut}_{E_{\infty}}(S),
$$

the map $f$ is weakly equivalent to a unique replacement map $R^{\prime} \rightarrow S^{\prime}$ of $G$-equivariant $E_{\infty}$-ring spectra. Taking $f$ to be the identity map of $R$ proves the first statement of the lemma; taking $f$ to be arbitrary proves the second.

Having fixed a base map $f$, the space of based maps $S^{n} \rightarrow \operatorname{Map}_{E_{\infty}}(R, S)$ is identified by adjunction with the space of $E_{\infty}$-maps which are lifts in the diagram

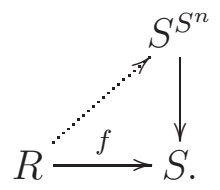


For $n>0$, the multiplication-by- $|G|$ map on $S^{n}$ induces an $E_{\infty}$ self-equivalence of $S^{S^{n}}$. Passing to $\pi_{0}$, we find that $g \mapsto g^{|G|}$ is a bijective self-map of $\pi_{n}\left(\operatorname{Map}_{E_{\infty}}(R, S), f\right)$. Therefore, the Mayer-Vietoris sequence implies that $\pi_{0} \mathrm{hAut}_{E_{\infty}}(f)$ is an extension of $\pi_{0} \mathrm{hAut}_{E_{\infty}}(R) \times_{\pi_{0} \operatorname{Map}_{E_{\infty}}(R, S)} \pi_{0} \mathrm{hAut}_{E_{\infty}}(S)$ by an abelian group in which multiplication by $|G|$ is an isomorphism. This implies that the given homomorphism from $G$ to the pullback on $\pi_{0}$, representing actions of $G$ on $R$ and $S$ commuting with $f$ in the homotopy category, lifts uniquely to a homomorphism

$$
G \rightarrow \pi_{0} \operatorname{hAut}_{E_{\infty}}(f) .
$$

Upon applying classifying spaces of the associated monoids, we obtain a diagram of spaces as follows:

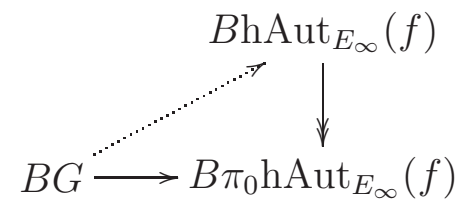

We now prove that there is a unique lift in the above diagram up to homotopy. The standard Postnikov-tower obstruction theory for lifting a map $Z \rightarrow X$ along a map $Y \rightarrow X$ gives an iterative sequence of obstructions in $H^{s}\left(Z ; \pi_{s}(X, Y)\right)$, with choices of lifts determined by classes in $H^{s}\left(Z ; \pi_{s+1}(X, Y)\right)$. In the present case, the homotopy groups of $B$ hAut $E_{\infty}(f)$ relative to $B \pi_{0}$ hAut $_{E_{\infty}}(f)$ are $p$-local in degrees above 1 , and so the cohomology of $B G$ vanishes; this implies the existence of a unique lift of $f$ to an equivariant map.

Given this lift, we can apply Cooke's techniques to replace the map $f: R \rightarrow S$ by a weakly equivalent map in the homotopy category such that $G$ acts on $R$ and $S$ in a way making the map $f G$-equivariant, as desired.

\section{$4 \quad K(2)$-local realization}

In this section we apply the Goerss-Hopkins-Miller theorem to show that any realization problem, as in Definition 3.1, has an essentially unique $K(2)$-local solution. 
Let $\zeta$ be a primitive $\left(p^{2}-1\right)$ 'st root of unity in $\mathbb{F}_{p^{2}}$. We will also use $\zeta$ to denote its Teichmüller lift in the $p$-typical Witt ring $\mathbb{W}\left(\mathbb{F}_{p^{2}}\right) \cong \mathbb{Z}_{p}[\zeta]$. Let $\sigma$ denote the nontrivial element of the Galois group $\operatorname{Gal}\left(\mathbb{F}_{p^{2}} / \mathbb{F}_{p}\right)$.

Definition 4.1. Suppose $(A, \mathbb{G})$ is a realization problem, and let $v \in A_{2 p^{2}-2}$ be any lift of the canonical class $v_{2}$ (which is only well-defined mod $\left(p, v_{1}\right)$ ). The associated Lubin-Tate ring is defined to be the graded A-algebra

$$
B=A_{K(2)}[\zeta, u] /\left(u^{p^{2}-1}-v\right)
$$

Here $\left|u_{1}\right|=0$ and $|u|=2$.

The associated group $G$ is defined to be the semidirect product

$$
\left(\mathbb{F}_{p^{2}}^{\times}\right) \rtimes \operatorname{Gal}\left(\mathbb{F}_{p^{2}} / \mathbb{F}_{p}\right)
$$

formed with respect to the action of the Galois group on $\mathbb{F}_{p^{2}}^{\times}$. The group $G$ acts on $\zeta$ through the quotient $\operatorname{Gal}\left(\mathbb{F}_{p^{2}} / \mathbb{F}_{p}\right)$, and any element $x \in \mathbb{F}_{p^{2}}^{\times} \subseteq G$ fixes $\zeta$ and acts on $u$ by multiplication by the Teichmuller lift of $x$.

Remark 4.2. If we choose a lift of $v_{1}$-class to $A$, we obtain by Remark 3.5 an isomorphism

$$
B \cong \mathbb{W}\left(\mathbb{F}_{p^{2}}\right) \llbracket u_{1} \rrbracket\left[u^{ \pm 1}\right],
$$

where the $A$-algebra structure is determined by $v_{1} \mapsto u_{1} u^{p-1}, v_{2} \mapsto u^{p^{2}-1}$. The group $G$ then acts on the whole graded $\mathbb{Z}_{p}$-algebra $B$ as follows:

$$
\begin{aligned}
\zeta \cdot u_{1} & =\zeta^{p-1} u_{1} & \sigma \cdot u_{1} & =u_{1} \\
\zeta \cdot u & =\zeta^{-1} u & \sigma \cdot u & =u
\end{aligned}
$$

Remark 4.3. To justify referring to $B$ as a Lubin-Tate ring, we observe the following. The action of $G$ preserves the graded formal group law. By construction, the ungraded quotient ring $B /(u-1)$ is the complete local ring $\mathbb{W}\left(\mathbb{F}_{p^{2}}\right) \llbracket u_{1} \rrbracket$ with maximal ideal $\left(p, u_{1}\right)$. Modulo $p$, the image of the formal group law $\mathbb{G}$ of $B$ has $p$-series congruent to $u_{1} x^{p}$ plus higher order terms, and modulo $\left(p, u_{1}\right)$ it has $p$-series congruent to $x^{p^{2}}$ plus higher order terms. Hence the image of $\mathbb{G}$ over $B$ is of exact height 2, carries a canonical choice of invariant differential, and is a universal deformation of the reduction of this data to $\mathbb{F}_{p^{2}}$ [LT66, Proposition 1.1]. 
Lemma 4.4. Suppose $(A, \mathbb{G})$ is a realization problem, $v \in A_{2 p^{2}-2}$ a lift of $v_{2}$, and $B$ the associated Lubin-Tate ring with associated group $G$. Then $B$ is a Galois extension of $A_{K(2)}$ with Galois group $G$, in the sense of Rog08, Definition 2.3.1].

Proof. The ring $B$ is the extension of $A_{K(2)}$ obtained by adjoining the primitive $\left(p^{2}-1\right)$ 'st root of unity $\zeta$ and the element $u$ satisfying $u^{p^{2}-1}=v$. The underlying module of $B$ is finitely generated free as a module over $A_{K(2)}$, with basis given by the images of the element $\sum_{i=0}^{p^{2}-2} \zeta u^{i}$ under the action of G. Therefore,

$$
B \cong A_{K(2)} \otimes \mathbb{Z}[G]
$$

as a module with $G$-action. The fixed subring is $A_{K(2)}$, and the map

$$
B \otimes_{A_{K(2)}} B \rightarrow \prod_{G} B
$$

is an isomorphism, as desired.

Theorem 4.5. Suppose $(A, \mathbb{G})$ is a realization problem. Then there exists a solution to the realization problem for the A-algebra $A_{K(2)}$ by a $K(2)$-local $E_{\infty}$-ring spectrum $R_{K(2)}$, and any two solutions to the realization problem for $A_{K(2)}$ are equivalent.

Proof. We first show that a solution exists. Let $v \in A_{2 p^{2}-2}$ be any lift of the canonical class $v_{2}$ as in Definition 4.1. Let $B$ be the associated Lubin-Tate ring and $G$ the associated group.

Remark 4.3, together with the Goerss-Hopkins-Miller theorem GH04, Corollary 7.6], imply that there exists a Lubin-Tate spectrum $E$ with $E_{*} \cong B$ in a manner preserving the associated formal group laws: the formal group law of $B$ lifts to an orientation $M U \rightarrow E$. This spectrum $E$ has an essentially unique structure as an $E_{\infty}$-ring spectrum, and the group $G$ acts on $E$ by maps of $E_{\infty}$-ring spectra. We therefore have a homotopy fixed point spectrum $E^{h G}$ which is an $E_{\infty}$-ring spectrum. We define $R_{K(2)}$ to be $E^{h G}$, and remark that $R_{K(2)}$ is $K(2)$-local since $E$ is. We will now show that it is a solution to the realization problem for $A_{K(2)}$. 
Lemma 4.4 implies that the fixed subring of $E_{*}$ under the action of $G$ is isomorphic to $A_{K(2)}$, and that the homotopy fixed point spectral sequence

$$
H^{s}\left(G ; \pi_{t} E\right) \Rightarrow \pi_{t-s} R_{K(2)}
$$

degenerates to an isomorphism

$$
\pi_{*}\left(R_{K(2)}\right) \cong A_{K(2)}
$$

The orientation $M U_{*} \rightarrow E_{*}$ factors through $\pi_{*}\left(R_{K(2)}\right) \cong A_{K(2)}$ because the graded formal group law of $E_{*}$ arises by base change from $A$ (and hence from $\left.A_{K(2)}\right)$. Therefore, Corollary 3.8 implies that there exists a corresponding lift of the orientation to an orientation $M U \rightarrow R_{K(2)}$. We thus find that $R_{K(2)}$ solves the realization problem for $A_{K(2)}$.

We now show that any other solution is equivalent, and in particular that the solution is independent of $v$. Suppose $T$ is another solution to the realization problem for $A_{K(2)}$. By Lemma 4.4 the map $T_{*} \cong A_{K(2)} \rightarrow B$ is a Galois extension with Galois group $G$, and in particular étale. Therefore, the results of [BR07, Section 2] imply that there exists a unique $E_{\infty} T$-algebra $E^{\prime}$ inducing this map on homotopy groups. As the map $T \rightarrow E^{\prime}$ is Galois on homotopy groups, we have a weak equivalence

$$
E^{\prime} \underset{T}{\wedge} E^{\prime} \rightarrow \prod_{g \in G} E^{\prime}
$$

Rognes' work on idempotents [Rog08, Section 10] shows that $E^{\prime} \wedge_{T} E^{\prime} \simeq$ $\prod_{G} E^{\prime}$ as a left $E^{\prime}$-algebra, and that the space of $T$-algebra maps $E^{\prime} \rightarrow E^{\prime}$, or equivalently the space of $E^{\prime}$-algebra maps $E^{\prime} \wedge_{T} E^{\prime} \rightarrow E^{\prime}$, is homotopically discrete and equivalent to $G$. Therefore, $E^{\prime}$ may be replaced by an equivalent $T$-algebra with a genuine action of $G$.

Moreover, $E^{\prime}$ is a Lubin-Tate spectrum, and the Goerss-Hopkins-Miller theorem implies that the $G$-equivariant identification of $\pi_{*} E$ with $\pi_{*} E^{\prime}$ lifts to a $G$-equivariant equivalence of $E_{\infty}$-ring spectra $E \rightarrow E^{\prime}$. The induced equivalence of homotopy fixed point spectra $E^{h G} \rightarrow\left(E^{\prime}\right)^{h G}$ is then an equivalence of $E_{\infty}$-ring spectra $R_{K(2)} \rightarrow T$ respecting the identification on the level of underlying homotopy groups. By definition, this means the two solutions $R_{K(2)}$ and $T$ are equivalent, as desired. 


\section{$5 \quad K(1)$-local obstruction theory}

The work in this section closely follows [Beh, Section 7], which in turn follows [GH, Section 2.2]. For background on $K(1)$-local $E_{\infty}$-ring spectra the reader may consult Hop and Lau03.

The aim is to explain in some generality the construction of $K(1)$-local $E_{\infty^{-}}$ ring spectra from purely algebraic data. We will always be working locally at some fixed prime $p$.

Section 5.1 records some basic properties of the $K(n)$-local Adams-Novikov spectral sequence.

Section 5.2 describes the structure supported by the $K$-theory of a $K(1)$ local ring spectrum. The reader should consult [Goe, Section 7.4] for a more conceptional explanation of some of these results.

Section 5.3 addresses a slightly technical issue which can best be explained in the context of realizing families of Landweber exact theories as in Goe09. We need to construct realizations not only over schemes of the form $\operatorname{Spec}(A)$, but slightly more generally over quotients of affine schemes by an action of $\mathbb{Z} /(p-1)$. To do so, we will need to rigidify the formal group that appears, and this is done by means of the Igusa tower.

The final section 5.4 contains the key constructive result in the $K(1)$-local setting (Theorem 5.20) which produces the desired $K(1)$-local $E_{\infty}$-ring spectrum and its chromatic attaching map.

We will write $K$ for the complex $K$-theory spectrum. Following [Hov08], we write $K_{*}^{\vee}(X)$ for $\pi_{*} L_{K(1)}(K \wedge X)$; this is isomorphic to $\pi_{*} \operatorname{holim}\left(K / p^{n} \wedge X\right)$ for $K / p^{n}$ denoting the smash product of $K$ with a $\bmod p^{n}$ Moore spectrum. We write $K_{p}^{\wedge}$ for $L_{K(1)} K$ itself.

\subsection{The $K(n)$-local Adams-Novikov spectral sequence}

Theorem 5.1. Recall that a prime $p$ is fixed, and choose an integer $n \geq 1$. Let $E$ denote the standard Lubin-Tate spectrum of height $n, K(n)$ the corresponding Morava $K$-theory, and $\mathbb{G}_{n}$ the extended Morava stabilizer group. For every $K(n)$-local spectrum $X$, there is a $K(n)$-localized E-based Adams- 
Novikov spectral sequence, converging strongly to the homotopy of $X$. If $E_{*} X$ is a flat $E_{*}$-module, this spectral sequence is of the form

$$
E_{2}^{s, t}=H_{c}^{s}\left(\mathbb{G}_{n} ; E_{t}^{\vee} X\right) \Rightarrow \pi_{t-s} X
$$

Here $E_{*}^{\vee} X=\pi_{*} L_{K(n)}(E \wedge X)$ is the Morava module of $X$. This spectral sequence is natural in $X$.

Remark 5.2. In this paper, we will only use the case $n=1$. Here we have $\mathbb{G}_{1}=\mathbb{Z}_{p}^{\times}, E=K_{p}^{\wedge}$ is $p$-adic $K$-theory, and $E_{*} X$ is flat if and only if it is $p$-torsion free.

Proof. The spectral sequence is constructed in [DH04, Appendix A] (with $Z=S^{0}$ ) and the convergence established in [DH04, Proposition A.3]. We need to determine the $E_{2}$-term, which by [DH04, Remark A.9] is the cohomology of the complex associated to the cosimplicial abelian group

$$
\pi_{*} L_{K(n)}\left(E^{(\bullet+1)} \wedge X\right) .
$$

By Landweber exactness of $E$ and our flatness assumption, we know that the $E_{*}$-module

$$
\pi_{*}\left(E^{(\bullet+1)} \wedge X\right) \cong \underbrace{E_{*} E \otimes_{E_{*}} \cdots \otimes_{E_{*}} E_{*} E}_{\bullet \text { times }} \otimes_{E_{*}} E_{*} X
$$

is flat as a left $E_{*}$-module. Application of [Hov, Theorem 3.1] with $M=$ $E^{(\bullet+1)} \wedge X$, along with Morava's fundamental computation ([DH04, Proposition 2.2], see in particular page 9 ), shows that

$$
\pi_{*} L_{K(n)}\left(E^{(\bullet+1)} \wedge X\right) \cong \operatorname{Map}_{c}\left(\left(\mathbb{G}_{n}\right)^{\bullet}, E_{*}\right) \widehat{\otimes}_{E_{*}} E_{*}^{\vee} X .
$$

In addition, we conclude that $E_{*}^{\vee} X \cong\left(E_{*} X\right)_{\mathfrak{m}}^{\wedge}$ for $\mathfrak{m} \subseteq E_{*}$ the unique homogeneous maximal ideal. Because $\mathbb{G}_{n}$ is profinite and $E_{*}^{\vee} X$ is m-adically complete, the canonical map

$$
\operatorname{Map}_{c}\left(\left(\mathbb{G}_{n}\right)^{\bullet}, E_{*}\right) \widehat{\otimes}_{E_{*}} E_{*}^{\vee} X \rightarrow \operatorname{Map}_{c}\left(\left(\mathbb{G}_{n}\right)^{\bullet}, E_{*}^{\vee} X\right)
$$

is an isomorphism. This identifies the $E_{1}$-term as the standard complex computing continous group cohomology, as claimed.

Remark 5.3. Further results on the identification of the $E_{2}$-term of this spectral sequence may be found in [Dav06, Theorem 10.2] and [DT]. 


\section{$5.2 \quad K(1)$-local complex oriented theories}

Denote by $M U P$ the 2-periodic version of the complex bordism spectrum $M U$.

Lemma 5.4. Suppose $F$ is a complex oriented, homotopy commutative ring spectrum such that $F_{*}$ is p-torsion free. Then there is a natural isomorphism

$$
\left(K_{*} \otimes_{M U_{*}} M U_{*} M U \otimes_{M U_{*}} F_{*}\right)_{p}^{\wedge} \rightarrow K_{*}^{\vee} F
$$

If $F$ is also even-periodic and equipped with an MUP-orientation, then there is also a natural isomorphism

$$
\left(K_{0} \otimes_{M U P_{0}} M U P_{0} M U P \otimes_{M U P_{0}} F_{0}\right)_{p}^{\wedge} \rightarrow K_{0}^{\vee} F
$$

Proof. As $F$ is complex orientable, the natural map

$$
M U_{*} M U \otimes_{M U_{*}} F_{*} \rightarrow M U_{*} F
$$

is an isomorphism. In particular, as $M U_{*} M U$ is a free right $M U_{*}$-module, both sides are isomorphic to a direct sum of copies of $F_{*}$ and hence are torsion-free. Smashing with a mod $p^{n}$-Moore spectrum $M\left(p^{n}\right)$, we obtain an inverse system of isomorphisms

$$
\left(M U_{*} M U \otimes_{M U_{*}} F_{*}\right) / p^{n} \rightarrow M U_{*}\left(M\left(p^{n}\right) \wedge F\right)
$$

as $n$ ranges over the natural numbers. Applying $K_{*} \otimes_{M U_{*}}-$, the ConnerFloyd isomorphism then implies that the natural map

$$
\left(K_{*} \otimes_{M U_{*}} M U_{*} M U \otimes_{M U_{*}} F_{*}\right) / p^{n} \rightarrow\left(K / p^{n}\right)_{*}(F)
$$

is an isomorphism. Taking the inverse limit over $n$, we obtain the desired result because the torsion-freeness of $K_{*} F$ implies

$$
K_{*}^{\vee} F \cong\left(K_{*} F\right)_{p}^{\wedge} \cong \lim _{n}\left(K / p^{n}\right)_{*}(F)
$$

If $F$ is also even-periodic, the above proof then carries through with $M U$ replaced by $M U P$. Taking the term of degree zero gives the desired result. 
We refer to [Str99a, Examples 2.6 and 2.9] for the universal properties of the two canonical ring homomorphisms $M U P_{0} \rightarrow M U P_{0} M U P$ employed in the proof of the following lemma.

Lemma 5.5. Suppose $T$ is a p-adic ring equipped with a formal group law $\mathbb{G}$, induced by a map $M U P_{0} \rightarrow T$, such that the induced formal group law on $T / p$ is of exact height 1 . Define

$$
V=\left(K_{0} \otimes_{M U P_{0}} M U P_{0} M U P \otimes_{M U P_{0}} T\right)_{p}^{\wedge}
$$

Then:

1. The extension $T \rightarrow V$ has a canonical structure of an ind-Galois extension with Galois group $\mathbb{Z}_{p}^{\times}$. In particular, $V$ is the p-adic completion of a filtered direct system of finite Galois extensions of $T$.

2. For all continuous characters $\chi: \mathbb{Z}_{p}^{\times} \rightarrow \mathbb{Z}_{p}^{\times}$, the twisted $\mathbb{Z}_{p}^{\times}$-module $V(\chi)$ is cohomologically trivial: for all closed subgroups $H<\mathbb{Z}_{p}^{\times}$, the continuous Galois cohomology $H_{c}^{s}(H, V(\chi))$ vanishes for $s>0$.

3. If $T$ is the p-adic completion of a smooth $\mathbb{Z}_{p}$-algebra, then $V$ is the p-adic completion of an ind-smooth $\mathbb{Z}_{p}$-algebra: $V$ is the p-adic completion of a filtered direct system of smooth $\mathbb{Z}_{p}$-algebras.

Remark 5.6. A mod- $p$ version of part 1 of this result, and a generalization to higher height, appear in [Bak01, Theorem A.1 and Theorem A.3].

Proof. The universal properties of $M U P_{0}$ and $M U P_{0} M U P$ imply that the ring map $T \rightarrow V$ is initial among continuous maps of $p$-adic rings $f: T \rightarrow S$ equipped with an isomorphism $f^{*} \mathbb{G} \rightarrow \widehat{\mathbb{G}}_{m}$.

Consequently, $V$ is the $p$-completion of the directed limit of the $T$-algebras $V_{n}$, where $T \rightarrow V_{n}$ is initial among continuous maps of $p$-adic rings $f: T \rightarrow S$ equipped with an isomorphism $f^{*} \mathbb{G}\left[p^{n}\right] \rightarrow \mu_{p^{n}}=\widehat{\mathbb{G}}_{m}\left[p^{n}\right]$.

By Cartier duality [Tat67, Section 1.2], these are equivalent to isomorphisms $\mathbb{Z} / p^{n} \rightarrow f^{*} \mathbb{G}^{\vee}\left[p^{n}\right]$ between the dual groups, or equivalently elements of $\mathbb{G}^{\vee}$ of order exactly $p^{n}$. As $\mathbb{G}$ is of height 1 and dimension 1 , the group scheme $\mathbb{G}^{\vee}\left[p^{n}\right]$ is étale-locally isomorphic to $\mathbb{Z} / p^{n}$. Therefore, the subscheme $\operatorname{Spf}\left(V_{n}\right)$ of generators is a principal $\left(\mathbb{Z} / p^{n}\right)^{\times}$-torsor over $\operatorname{Spf}(T)$. The maps 
$\operatorname{Spf}\left(V_{n+1}\right) \rightarrow \operatorname{Spf}\left(V_{n}\right)$ are the restrictions of the multiplication-by- $p$ maps $\mathbb{G}^{\vee}\left[p^{n+1}\right] \rightarrow \mathbb{G}^{\vee}\left[p^{n}\right]$, and therefore the pro-system $\operatorname{Spf}\left(V_{n}\right)$ assembles into a principal torsor for the constant pro-group scheme $\mathbb{Z}_{p}^{\times}$.

However, by definition this is the same as the map $T \rightarrow V$ being an ind-Galois extension of $p$-adic rings with Galois group $\mathbb{Z}_{p}^{\times}$, establishing item 1 ,

Now fix a continuous character $\chi: \mathbb{Z}_{p}^{\times} \rightarrow \mathbb{Z}_{p}^{\times}$. Recall that the twisted $\mathbb{Z}_{p}^{\times}$module $V(\chi)=V \otimes_{\mathbb{Z}_{p}} \mathbb{Z}_{p}(\chi)$ has underlying $\mathbb{Z}_{p}$-module $V$ but $\mathbb{Z}_{p}^{\times}$-action given by

$$
\alpha \cdot v=\chi(\alpha)(\alpha v)
$$

for all $\alpha \in \mathbb{Z}_{p}^{\times}, v \in V$.

Arguing as in [Tat76, Proposition 2.1 and p. 259], one reduces the proof of cohomological triviality for $V(\chi)$ to that for the module

$$
\overline{V(\chi)}:=V(\chi) / p V(\chi) \text {. }
$$

If $\chi$ is the trivial character, the result is then immediate from $V / p V$ being a $\mathbb{Z}_{p}^{\times}$-Galois extension of $T / p$. For general $\chi$, we have $\overline{V(\chi)}=(V / p V)(\bar{\chi})$ where $\bar{\chi}$ is the composite

$$
\bar{\chi}: \mathbb{Z}_{p}^{\times} \stackrel{\chi}{\longrightarrow} \mathbb{Z}_{p}^{\times} \longrightarrow(\mathbb{Z} / p)^{\times} .
$$

For the subgroup $U=H \cap\left(1+p \mathbb{Z}_{p}\right) \subseteq \mathbb{Z}_{p}^{\times}$we have $\overline{V(\chi)} \cong V / p V$ as $U$-modules, and this is cohomologically trivial over $U$ by the above. One concludes cohomological triviality over $H$ by a straightforward application of the Lyndon-Hochschild-Serre spectral sequence to the exact sequence $1 \rightarrow$ $U \rightarrow H \rightarrow H / U \rightarrow 1$ because $H / U$ has order prime to $p$. This shows item 2 ,

To see item 3, suppose in addition that $T$ is the $p$-adic completion of a smooth $\mathbb{Z}_{p}$-algebra. For every $n \geq 1$, the fact that $T \subseteq V_{n}$ is finite étale implies that $V_{n}$, too, is the $p$-adic completion of a smooth $\mathbb{Z}_{p}$-algebra. Hence

$$
\mathbb{Z}_{p} \rightarrow \bigcup_{n \geq 1} V_{n}
$$

is a filtered direct limit (in fact, an increasing union) of $p$-adic completions of smooth $\mathbb{Z}_{p}$-algebras, and we argued previously that $V=\left(\cup_{n \geq 1} V_{n}\right)_{p}^{\wedge}$. 
Corollary 5.7. Suppose $F$ is a $K(1)$-local, complex oriented, even-periodic homotopy commutative ring spectrum with $F_{*}$ torsion-free such that $F_{0}$ is the p-adic completion of a smooth $\mathbb{Z}_{p}$-algebra. Then the ring $K_{0}^{\vee} F$ is the p-adic completion of an ind-smooth $\mathbb{Z}_{p}$-algebra, as well as the p-adic completion of an ind-étale $F_{0}$-algebra.

Proof. By Lemma 5.4, we have

$$
K_{0}^{\vee} F \cong\left(\mathbb{Z} \otimes_{M U P_{0}} M U P_{0} M U P \otimes_{M U P_{0}} F_{0}\right)_{p}^{\wedge}
$$

We obtain a composite map

$$
\mathbb{Z}_{p} \rightarrow F_{0} \rightarrow K_{0}^{\vee} F
$$

where $F_{0}$ is the $p$-adic completion of a smooth $\mathbb{Z}_{p}$-algebra by assumption. The result now follows from Lemma 5.5 with $T=F_{0}$, remarking that $V=K_{0}^{\vee} F$ by Lemma 5.4 and that the formal group law is of strict height 1 by the assumption that $F$ is $K(1)$-local.

The main point of the next result is to weaken the assumption that $F_{*}$ be even-periodic. We remark that item 4 of it is a special case of [DT, Theorem $1.3]$.

Corollary 5.8. Suppose $F$ is a $K(1)$-local, complex oriented, homotopy commutative ring spectrum with associated formal group law $\mathbb{G} / F_{*}$ such that $F_{*}$ is p-torsion free and concentrated in even degrees. Then the following consequences hold.

1. The spectrum $F$ is $(2 p-2)$-periodic.

2. The map $F_{*} \rightarrow\left(K_{*}^{\vee} F\right)^{\mathbb{Z}_{p}^{\times}}$is an isomorphism.

3. For all closed subgroups $H$ of $\mathbb{Z}_{p}^{\times}$, the continuous Galois cohomology groups $H_{c}^{s}\left(H, K_{t}^{\vee} F\right)$ vanish for all $t \in \mathbb{Z}$ and all $s>0$.

4. The natural map $F \rightarrow\left(L_{K(1)}(K \wedge F)\right)^{h \mathbb{Z}_{p}^{\times}}$is a weak equivalence. 
Proof. We note that the orientation of $F$ provides a map $L_{K(1)} M U \rightarrow F$ of ring spectra. However, the localization map $M U \rightarrow v_{1}^{-1} M U$ is a $K(1)$ equivalence, and so $F_{*}$ is an algebra over $v_{1}^{-1} M U_{*}$. This ring is therefore $(2 p-2)$-periodic, as in item 11. The choice of a periodicity generator $v_{1} \in$ $F_{2 p-2}$ lets us introduce $F_{*}^{\text {per }}=F_{*} /\left(v_{1}-1\right)$, an associated $\mathbb{Z} /(2 p-2)$-graded $p$-adic ring.

By Lemma 5.4, the ring

$$
\left(K_{*}^{\vee} F\right)^{p e r} \cong\left(\left(K_{*} \otimes_{M U_{*}} M U_{*} M U \otimes_{M U_{*}} F_{*}\right)_{p}^{\wedge}\right)^{p e r}
$$

parameterizes strict isomorphisms between the formal group law $\mathbb{G}$ and formal group laws of the form $x+y-\beta x y$, for $\beta$ invertible, over $p$-adic $F_{*}^{p e r}$ algebras. As each such formal group law admits the canonical nonstrict isomorphism $x \mapsto \beta x$ to the multiplicative formal group law, this is equivalent to parameterizing nonstrict isomorphisms between the multiplicative formal group law and the formal group law of $F_{*}^{p e r}$. As such, if we identify the map $M U_{*} \rightarrow F_{*}^{p e r}$ with the corresponding map of ungraded rings $M U P_{0} \rightarrow F_{*}^{p e r}$, we obtain an isomorphism of ungraded rings

$$
\left(K_{*}^{\vee} F\right)^{p e r} \cong\left(\mathbb{Z} \otimes_{M U P_{0}} M U P_{0} M U P \otimes_{M U P_{0}} F_{*}^{p e r}\right)_{p}^{\wedge}
$$

Observe $T:=F_{*}^{p e r}$ is a $p$-adic ring because $F$ is $K(1)$-local and $F_{*}$ is torsionfree. Applying Lemma 5.5 to $T=F_{*}^{\text {per }}$ and extrapolating back to $F_{*}$, we find that $F_{*}$ is the ring of $\mathbb{Z}_{p}^{\times}$-invariants in $K_{*}^{\vee} F$ (item 2 holds true) and that all twists of the $\mathbb{Z}_{p}^{\times}$-module $K_{0}^{\vee} F$ are cohomologically trivial. Item 3 now follows because for every $t \in \mathbb{Z}$, the $\mathbb{Z}_{p}^{\times}$-module $K_{t}^{\vee} F$ is either zero or the twist of $K_{0}^{\vee} F=V$ by the action of $\mathbb{Z}_{p}^{\times}$on the $(t / 2)^{\prime}$ th power of the Bott element. The final item 4 follows from items 2 and 3 and the Adams-Novikov spectral sequence in Theorem 5.1.

Remark 5.9. We note that switching to $(2 p-2)$-periodic rings in the previous proof is solely to avoid a technical issue. Namely, the underlying ring of a $p$-adic $\mathbb{Z}$-graded ring is not $p$-adic when considered as an ungraded ring, but the analogous assertion does hold true for $(2 p-2)$-periodic rings.

\section{3 $\quad K(1)$-local theories and periodicity}

In order to translate results about even-periodic cohomology theories into results about general $K(1)$-local complex orientable theories, we recall the 
following construction. For every $n \geq 1$, we have an exact sequence

$$
0 \rightarrow\left(1+p^{n} \mathbb{Z}_{p}\right) \rightarrow \mathbb{Z}_{p}^{\times} \rightarrow\left(\mathbb{Z} / p^{n}\right)^{\times} \rightarrow 0
$$

of groups, and $\mathbb{Z}_{p}^{\times}$acts by $E_{\infty}$-ring maps on the $p$-adic $K$-theory spectrum $K_{p}^{\wedge}$.

Definition 5.10. For every $n \geq 1$, let

$$
\mathbb{S}\left(p^{n}\right)=\left(K_{p}^{\wedge}\right)^{h\left(1+p^{n} \mathbb{Z}_{p}\right)}
$$

be the $K(1)$-local $E_{\infty}$-ring spectrum which is the continuous homotopy fixed point object of $\left(1+p^{n} \mathbb{Z}_{p}\right)$ acting on $K_{p}^{\wedge}$, constructed by Devinatz and Hopkins [DH04]. This is equipped with an action of $\left(\mathbb{Z} / p^{n}\right)^{\times}$. If $F$ is a $K(1)$-local spectrum, then we define $F\left(p^{n}\right)$ to be the $K(1)$-local smash product

$$
L_{K(1)}\left(F \wedge \mathbb{S}\left(p^{n}\right)\right),
$$

viewed as a $\left(\mathbb{Z} / p^{n}\right)^{\times}$-spectrum. We refer to the resulting tower of $K(1)$-local spectra

$$
F \rightarrow F(p) \rightarrow F\left(p^{2}\right) \rightarrow \cdots
$$

as the Igusa tower of $F$.

The nomenclature in Definition 5.10 is motivated by item 2 in the following result.

Lemma 5.11. Suppose $F$ is a $K(1)$-local, complex oriented, homotopy commutative ring spectrum with associated formal group law $\mathbb{G} / F_{*}$ such that $F_{*}$ is $p$-torsion free and concentrated in even degrees, and let $n \geq 1$.

1. The spectrum $F\left(p^{n}\right)$ is an $F$-module spectrum. If $F$ is an $E_{\infty}$-ring spectrum, then $F\left(p^{n}\right)$ is also canonically an $E_{\infty}$-ring spectrum and the group $\left(\mathbb{Z} / p^{n}\right)^{\times}$acts on $F\left(p^{n}\right)$ by maps of $E_{\infty} F$-algebras.

2. The $F_{*}$-algebra $F\left(p^{n}\right)_{*}$ is the coordinate ring $\mathcal{O}_{\mathbb{G}^{\vee}\left[p^{n}\right] \backslash \mathbb{G}^{\vee}\left[p^{n-1}\right]}$ of the elements of exact order $p^{n}$ in the $p$-divisible group $\mathbb{G}^{\vee}$ which is Serre dual to $\mathbb{G}$. (See Tat67, Section 2.3] for duality of p-divisible groups.)

3. The canonical map $F \rightarrow F\left(p^{n}\right)^{h\left(\mathbb{Z} / p^{n}\right)^{\times}}$is a homotopy equivalence. 
4. The spectrum $F(p)$ is even-periodic, and has the homotopy type of $\bigvee_{k=0}^{p-2} \Sigma^{2 k} F$. Specifically, we have $F(p)_{*} \cong F_{*}[s] /\left(s^{p-1}-b\right)$ as graded $F_{*}$-algebras for a suitable unit $b \in F_{2-2 p}$.

Proof. Item 1 follows from the fact that the group $\mathbb{Z}_{p}^{\times}$acts on the $p$-adic $K$ theory spectrum by $E_{\infty}$-ring maps, and therefore $\mathbb{S}\left(p^{n}\right)$ inherits a $\left(\mathbb{Z} / p^{n}\right)^{\times}$equivariant $E_{\infty}$-ring structure.

To establish item 2, recall that we have just seen that the ring $F\left(p^{n}\right)_{*}$ is the subring of $\left(1+p^{n} \mathbb{Z}_{p}\right)$-invariants in the graded ring $K_{*}^{\vee} F$. As in the proof of Corollary 5.8, after periodification $K_{*}^{\vee} F$ is the universal ring parameterizing nonstrict isomorphisms between the multiplicative formal group law and $\mathbb{G}$ over $p$-adic $F_{*}$-algebras. The isomorphism $\mathbb{G}\left[p^{n}\right] \rightarrow \mu_{p^{n}}$ obtained by restricting the universal isomorphism $\mathbb{G} \cong \widehat{\mathbb{G}}_{m}$ over $K_{*}^{\vee} F$ is invariant under the action of $\left(1+p^{n} \mathbb{Z}_{p}\right)$ and hence defined over $F\left(p^{n}\right)_{*}$. Moreover, the ring parameterizing isomorphisms $\mathbb{G} \rightarrow \widehat{\mathbb{G}}_{m}$ which restrict to this given isomorphism is a principal $\left(1+p^{n} \mathbb{Z}_{p}\right)$-torsor over $F_{*}$. The ring of invariants $F\left(p^{n}\right)_{*}$ is precisely the universal $F_{*}$-algebra parameterizing isomorphisms $\mathbb{G}\left[p^{n}\right] \rightarrow \mu_{p^{n}}$ of finite flat group schemes, and the grading is recovered through the action of nonstrict isomorphisms $x \mapsto u x$.

As above, isomorphisms $\mathbb{G}\left[p^{n}\right] \rightarrow \mu_{p^{n}}$ are in functorial bijection with isomorphisms $\mathbb{Z} / p^{n} \rightarrow \mathbb{G}^{\vee}\left[p^{n}\right]$ by Cartier duality [Tat67, Section 1.2]. The finite group scheme $\mathbb{G}^{\vee}\left[p^{n}\right]$ is étale-locally cyclic of order $p^{n}$, and thus such an isomorphism is equivalent to a choice of a section of $\mathbb{G}^{\vee}\left[p^{n}\right] \backslash \mathbb{G}^{\vee}\left[p^{n-1}\right]$. Therefore, the map $F_{*} \rightarrow F\left(p^{n}\right)_{*}$ is a $\left(\mathbb{Z} / p^{n}\right)^{\times}$-Galois extension with range isomorphic to the coordinate ring $\mathcal{O}_{\mathbb{G}^{\vee}}\left[p^{n}\right] \backslash \mathbb{G}^{\vee}\left[p^{n-1}\right]$, implying item 2. The fact that this extension is Galois implies that the homotopy fixed point spectral

$$
H^{s}\left(\left(\mathbb{Z} / p^{n}\right)^{\times} ; F\left(p^{n}\right)_{*}\right) \Rightarrow \pi_{*} F\left(p^{n}\right)^{h\left(\mathbb{Z} / p^{n}\right)^{\times}}
$$

degenerates, and the map $F \rightarrow F\left(p^{n}\right)^{h\left(\mathbb{Z} / p^{n}\right)^{\times}}$is a weak equivalence, establishing item 3 ,

For item 4, we employ the work of Oort-Tate [TO70] classifying finite flat group schemes of rank $p$ in order to determine the structure of the $F_{*}$-algebra $F(p)_{*}$. The coordinate ring

$$
\mathcal{O}_{\mathbb{G}[p]} \cong F_{*} \llbracket t \rrbracket /[p]_{\mathbb{G}}(t)
$$


is a finite free $F_{*}$-algebra of rank $p$ with basis $\left\{t^{k} \mid 0 \leq k<p\right\}$. The ring $\mathcal{O}_{\mathbb{G}[p]}$ has an action of $\mathbb{F}_{p}^{\times} \subseteq \mathbb{Z}_{p}^{\times}$given by $\zeta \cdot t=[\zeta]_{\mathbb{G}}(t)$, and without loss of generality we may replace the orientation class $t$ by a new generator $s$ of the power series ring such that $\zeta \cdot s=\zeta s$. We note that the action of nonstrict isomorphisms places the element $s$ in degree -2 .

The Oort-Tate classification implies that we have an isomorphism

$$
\mathcal{O}_{\mathbb{G}^{\vee}[p]} \cong \bigoplus_{k=0}^{p-1} I^{\otimes k}
$$

for $I$ an invertible $F_{*}$-module, dual to the submodule of $\mathcal{O}_{\mathbb{G}[p]}$ where $\mathbb{F}_{p}^{\times} \subseteq \mathbb{Z}_{p}^{\times}$ acts by scalar multiplication. In particular, the grading implies that $I$ is concentrated in degrees congruent to $-2 \bmod (2 p-2)$, so we must have $I$ free with generator $s$. The multiplication is induced by the tensor product pairing $I^{\otimes k} \otimes I^{\otimes l} \rightarrow I^{\otimes(k+l)}$ together with a map $I^{\otimes p} \rightarrow I$. The augmentation to $F_{*}$ sends $I$ to zero.

We find that the ring $\mathcal{O}_{\mathbb{G}^{\vee}[p]}$ is of the form

$$
F_{*}[s] /\left(s^{p}-b s\right),
$$

for some element $b \in F_{*}$, and the zero section corresponds to the quotient by the ideal $(s)$. As this scheme is étale, the Jacobian criterion Mil80, Chapter I, Example 3.4] implies that $b$ must be a unit in $F_{*}$.

Therefore, the coordinate ring of the complement of the zero section is

$$
F(p)_{*} \cong F_{*}[s] /\left(s^{p-1}-b\right) .
$$

As $b$ is invertible, this implies that $F(p)_{*}$ is 2-periodic. Moreover, the $F_{*}$ module $F(p)_{*}$ is free with basis $\left\{1, s, \ldots, s^{p-2}\right\}$. Choosing maps $S^{2 k} \rightarrow F(p)$ representing the elements $s^{k}$, the $F$-module structure from item 1 gives us maps $F \wedge S^{2 k} \rightarrow F(p)$ which together provide a weak equivalence $\bigvee \Sigma^{2 k} F \rightarrow$ $F(p)$ of $F$-modules. This completes the proof of item 4 .

Remark 5.12. Daniel Davis pointed out an error in a previous version which assumed a transitivity result for homotopy fixed points that is not implied by the work of Devinatz-Hopkins. A proof of this result will appear in forthcoming work of Davis and Torii. 
Corollary 5.13. Suppose $F$ is a $K(1)$-local, complex orientable, homotopy commutative ring spectrum such that $F_{*}$ is p-torsion free and concentrated in even degrees. If there exists a complex orientation $M U \rightarrow F(p)$ which, on homotopy groups, factors as $M U_{*} \rightarrow F_{*} \subseteq F(p)_{*}$, then there exists a complex orientation $M U \rightarrow F$ realizing the given map $M U_{*} \rightarrow F_{*}$.

Proof. Lemma 5.11, item 4 implies that $F(p)_{*}$ is faithfully flat over $F_{*}$, and then Corollary 3.8 shows that the orientation of $F(p)$ lifts to $F$.

\subsection{Obstruction theory}

For an $E_{\infty}$-ring spectrum $R$, the $p$-completed $K$-theory $K_{*}^{\vee} R$ has the structure of a $p$-complete ring equipped with an action of $\mathbb{Z}_{p}^{\times}$(the Adams operations $\psi^{n}$ for $\left.n \in \mathbb{Z}_{p}^{\times}\right)$by $\left(K_{p}^{\wedge}\right)_{*}$-algebra automorphisms, together with a power operation $\theta$ on $K_{0}^{\vee} R$ extending the operation of the same name on $R_{0}$ and commuting with the Adams operations. This operation satisfies $\psi^{p}(x)=x^{p}+p \theta(x)$. By contrast with the references, we will refer to such structure as a $\psi-\theta$-algebra, and reserve the term $\theta$-algebra for a $p$-adic ring equipped with the operation $\theta$ alone (such as the homotopy of a $K(1)$-local $K_{p}^{\wedge}$-algebra). These power operations were studied by McClure [BMMS86, Chapter IX]. See [GH, Section 2.2] for more details and the definition of the relevant version of André-Quillen cohomology, denoted here by $H_{\psi-\theta-a l g}^{*}$.

We recall the following consequences of the Goerss-Hopkins obstruction theory.

Theorem 5.14. 1. Given a graded $\psi-\theta$-algebra $A_{*}$, the obstructions to the existence of a $K(1)$-local $E_{\infty}$-ring spectrum $R$ for which there is an isomorphism

$$
K_{*}^{\vee} R \cong A_{*}
$$

of graded $\psi$ - $\theta$-algebras lie in

$$
H_{\psi-\theta-a l g}^{s}\left(A_{*} /\left(K_{p}^{\wedge}\right)_{*}, \Omega^{s-2} A_{*}\right), s \geq 3 .
$$

The obstructions to uniqueness lie in

$$
H_{\psi-\theta-a l g}^{s}\left(A_{*} /\left(K_{p}^{\wedge}\right)_{*}, \Omega^{s-1} A_{*}\right), s \geq 2 .
$$


2. Given $K(1)$-local $E_{\infty}$-ring spectra $R_{1}$ and $R_{2}$ such that $K_{*}^{\vee} R_{2}$ is $p$ complete, together with a map of graded $\psi-\theta$-algebras

$$
f_{*}: K_{*}^{\vee} R_{1} \rightarrow K_{*}^{\vee} R_{2}
$$

the obstructions to the existence of a map $f: R_{1} \rightarrow R_{2}$ of $E_{\infty}$-ring spectra inducing $f_{*}$ on p-adic K-theory lie in

$$
H_{\psi-\theta \text {-alg }}^{s}\left(K_{*}^{\vee} R_{1} /\left(K_{p}^{\wedge}\right)_{*}, \Omega^{s-1} K_{*}^{\vee} R_{2}\right), s \geq 2 .
$$

The obstructions to uniqueness lie in

$$
H_{\psi-\theta-a l g}^{s}\left(K_{*}^{\vee} R_{1} /\left(K_{p}^{\wedge}\right)_{*}, \Omega^{s} K_{*}^{\vee} R_{2}\right), s \geq 1 .
$$

3. Given $K(1)$-local $E_{\infty}$-ring spectra $R_{1}$ and $R_{2}$ and a fixed $E_{\infty}$-ring map $\phi: R_{1} \rightarrow R_{2}$ such that $K_{*}^{\vee} R_{2}$ is p-complete, there is a fringed secondquadrant spectral sequence with $E_{2}$-term

$$
E_{2}^{s, t}= \begin{cases}\operatorname{Hom}_{\psi-\theta-a l g}\left(K_{*}^{\vee} R_{1}, K_{*}^{\vee} R_{2}\right) & \text { for }(s, t)=(0,0), \\ H_{\psi-\theta \text {-alg }}^{s}\left(K_{*}^{\vee} R_{1} /\left(K_{p}^{\wedge}\right)_{*}, \Omega^{t} K_{*}^{\vee} R_{2}\right) & \text { for } t>0,\end{cases}
$$

abutting to

$$
\pi_{t-s}\left(\operatorname{Map}_{E_{\infty}}\left(R_{1}, R_{2}\right), \phi\right)
$$

In order, the references for the above facts are as follows:

1. This is an application of [GH04, Corollary 5.9] with the basic homology theory $E$ there being chosen as $p$-complete $K$-theory. To identify the resulting obstruction groups as $\psi-\theta$-cohomology, one invokes $\mathrm{GH}$, Sections 2.2 and 2.4].

2. [GH, Corollary 2.4.15], with $Z=S^{0}$.

3. [GH, Theorem 2.4.14], with $Z=S^{0}$.

The next result isolates purely algebraic assumptions which will demonstrate vanishing of many relevant André-Quillen cohomology groups.

Lemma 5.15. Suppose that $A_{*} \rightarrow B_{*}$ is a map of graded, p-adic, evenperiodic $\psi-\theta$-algebras concentrated in even degrees such that 
- $A_{0}$ is the p-adic completion of an ind-étale extension of the fixed subring $\left(A_{0}\right)^{\mathbb{Z}_{p}^{\times}} \subseteq A_{0}$,

- $\left(A_{0}\right)^{\mathbb{Z}_{p}^{\times}}$is the $p$-adic completion of a smooth $\mathbb{Z}_{p}$-algebra, and

- the continuous group cohomology $H_{c}^{s}\left(\mathbb{Z}_{p}^{\times}, \Omega^{t} B_{*}\right)$ vanishes for $s>0$ and all $t \in \mathbb{Z}$.

Then the $\psi$ - $\theta$-algebra cohomology

$$
H_{\psi-\theta-a l g}^{s}\left(A_{*} /\left(K_{p}^{\wedge}\right)_{*}, \Omega^{t} B_{*}\right)
$$

vanishes for $s \geq 2$ or $t$ odd.

Proof. To aid readability, define $T_{*}=\left(A_{*}\right)^{\mathbb{Z}_{p}^{\times}}$.

Using the smoothness assumption, we can apply [GH, Equation (2.4.9)], with $M=\Omega^{t} B_{*}$, to see that

$$
H_{\psi-\theta-a l g}^{s}\left(A_{*} /\left(K_{p}^{\wedge}\right)_{*}, \Omega^{t} B_{*}\right) \cong \operatorname{Ext}_{\operatorname{Mod}_{A_{*}}^{s-\theta}}\left(\Omega_{A_{*} /\left(K_{p}^{\wedge}\right)_{*}}, \Omega^{t} B_{*}\right) .
$$

As $A_{*}$ is even-periodic, sending a graded module $M_{*}$ to $\left(M_{0}, M_{1}\right)$ is an equivalence of categories between the category of graded $A_{*}-\psi-\theta$ modules and the category of pairs of $A_{0}-\psi-\theta$-modules. Therefore, there is an isomorphism of Ext groups

$$
\operatorname{Ext}_{M o d_{A_{*}}^{\psi^{-} \theta}}^{s}\left(\Omega_{A_{*} /\left(K_{p}^{\wedge}\right)_{*}}, \Omega^{t} B_{*}\right) \cong \operatorname{Ext}_{\operatorname{Mod}_{A_{0}}^{\psi^{-\theta}}}\left(\Omega_{A_{0} / \mathbb{Z}_{p}},\left(\Omega^{t} B\right)_{0}\right)
$$

because $A_{1}$ is trivial.

As $A_{0}$ is the $p$-adic completion of an ind-étale $T_{0}$-algebra, there is an isomorphism of modules of Kähler differentials $\Omega_{A_{0} / \mathbb{Z}_{p}} \cong A_{0} \otimes_{T_{0}} \Omega_{T_{0} / \mathbb{Z}_{p}}$ that respects the operation $\theta$ and the operations $\psi^{k}$ (the latter operations acting trivially on $T_{0}$ ). Since $A_{0}$ is flat over $T_{0}$, we get an isomorphism

$$
\operatorname{Ext}_{M o d_{A_{0}}^{\psi-\theta}}^{s}\left(\Omega_{A_{0} / \mathbb{Z}_{p}},\left(\Omega^{t} B\right)_{0}\right) \cong \operatorname{Ext}_{M o d_{T_{0}}^{\psi^{-}-}}^{s}\left(\Omega_{T_{0} / \mathbb{Z}_{p}},\left(\Omega^{t} B\right)_{0}\right) .
$$

The right-hand Ext-group is computed in the category of modules over $T_{0}$ equipped with a continuous $\mathbb{Z}_{p}^{\times}$-action and an operator $\theta$, and the $\mathbb{Z}_{p}^{\times}$-action is trivial on the domain. This leads to a composite functor spectral sequence:

$$
E_{2}^{s, u}=\operatorname{Ext}_{T_{0}[\theta]}^{s}\left(\Omega_{T_{0} / \mathbb{Z}_{p}}, H_{c}^{u}\left(\mathbb{Z}_{p}^{\times},\left(\Omega^{t} B\right)_{0}\right)\right) \Rightarrow \operatorname{Ext}_{M o d_{T_{0}}^{\psi-\theta}}^{s+u}\left(\Omega_{T_{0} / \mathbb{Z}_{p}},\left(\Omega^{t} B\right)_{0}\right)
$$


By assumption, the group cohomology degenerates, and we obtain the result

$$
H_{\psi-\theta-a l g}^{s}\left(A_{*} /\left(K_{p}^{\wedge}\right)_{*}, \Omega^{t} B_{*}\right) \cong \operatorname{Ext}_{T_{0}[\theta]}^{s}\left(\Omega_{T_{0} / \mathbb{Z}_{p}},\left(\Omega^{t} B\right)_{0}^{\mathbb{Z}_{p}^{\times}}\right) .
$$

By smoothness, $\Omega_{T_{0} / \mathbb{Z}_{p}}$ is a projective $T_{0}$-module, and hence these Ext-groups vanish for $s \geq 2$. They also vanish for $t$ odd because $B_{*}$ is concentrated in even degrees.

Direct application of Lemma 5.15 with $A_{*}=B_{*}$ and Theorem [5.14, item 11, yields the following existence and uniqueness result for $K(1)$-local $E_{\infty}$-ring spectra.

Corollary 5.16. Suppose that $A_{*}$ is an even-periodic graded p-adic $\psi-\theta$ algebra concentrated in even degrees such that

- $A_{0}$ is the p-adic completion of an ind-étale extension of $\left(A_{0}\right)^{\mathbb{Z}_{p}^{\times}}$,

- $\left(A_{0}\right)^{\mathbb{Z}_{p}^{\times}}$is the $p$-adic completion of a smooth $\mathbb{Z}_{p}$-algebra, and

- the continuous group cohomology $H_{c}^{s}\left(\mathbb{Z}_{p}^{\times}, \Omega^{t} A_{*}\right)$ vanishes for $s>0$ and all $t \in \mathbb{Z}$.

Then there exists a $K(1)$-local $E_{\infty}$-ring spectrum $R$ such that $K_{*}^{\vee} R \cong A_{*}$ as $\psi-\theta$-algebras, and any two such are weakly equivalent.

Remark 5.17. With the results from Section 5.2, the previous corollary establishes an equivalence between a homotopy category of certain $K(1)$-local $E_{\infty}$-ring spectra and a certain class of $\psi-\theta$-algebras.

Next, we start to study $E_{\infty}$-maps through purely algebraic data.

Proposition 5.18. Suppose that $F$ and $F^{\prime}$ are $K(1)$-local, even-periodic, complex oriented $E_{\infty}$-ring spectra such that the rings $F_{0}$ and $F_{0}^{\prime}$ are $p$-torsion free and such that $F_{0}$ is the p-adic completion of a smooth $\mathbb{Z}_{p}$-algebra. Then the canonical map

$$
[f] \mapsto K_{0}^{\vee}(f): \pi_{0} \operatorname{Map}_{E_{\infty}}\left(F, F^{\prime}\right) \rightarrow H_{o m}-\theta-a l g\left(K_{0}^{\vee} F, K_{0}^{\vee} F^{\prime}\right),
$$

from the connected components of the derived $E_{\infty}$-mapping space to the set of $\psi$ - $\theta$-algebra maps, is bijective. The canonical map

$$
[f] \mapsto \pi_{0}(f): \pi_{0} \operatorname{Map}_{E_{\infty}}\left(F, F^{\prime}\right) \rightarrow H_{o m}{ }_{\theta-a l g}\left(\pi_{0} F, \pi_{0} F^{\prime}\right)
$$


is injective. For any basepoint $f$ of the mapping space, the fundamental group $\pi_{1}\left(\operatorname{Map}_{E_{\infty}}\left(F, F^{\prime}\right), f\right)$ is abelian.

Proof. Define $A_{*}=K_{*}^{\vee} F$ and $B_{*}=K_{*}^{\vee} F^{\prime}$. By Corollary 5.7, the ring $A_{0}$ is the $p$-adic completion of an ind-étale $F_{0}$-algebra. By Corollary [5.8, items 2 and 3 , we have $F_{0}=\left(A_{0}\right)^{\mathbb{Z}_{p}^{\times}}$and $\left(F^{\prime}\right)_{0}=\left(B_{0}\right)^{\mathbb{Z}_{p}^{\times}}$. In addition, the cohomology groups $H_{c}^{s}\left(H, \Omega^{t} A_{*}\right)$ and $H_{c}^{s}\left(H, \Omega^{t} B_{*}\right)$ vanish for $H$ closed in $\mathbb{Z}_{p}^{\times}, s>0$ and all $t \in \mathbb{Z}$. Therefore, Lemma 5.15 implies that for any given map of $\psi-\theta$ algebras $A_{*} \rightarrow B_{*}$, the $\psi$ - $\theta$-algebra cohomology $H_{\psi-\theta \text {-alg }}^{s}\left(A_{*} /\left(K_{p}^{\wedge}\right)_{*}, \Omega^{t} B_{*}\right)$ vanishes for $s \geq 2$ or $t$ odd.

By Theorem 5.14, item 2 we therefore find that any map of $\psi$ - $\theta$-algebras $\bar{f}: A_{*} \rightarrow B_{*}$ has a lift to a map of $E_{\infty}$-ring spectra $f: F \rightarrow F^{\prime}$, unique up to homotopy. This is equivalent to the claimed bijectivity of the first displayed map in this proposition. By Theorem 5.14, item 3 the fundamental group of the mapping space is always abelian.

To address the injectivity of the map given by evaluating $\pi_{0}$, assume $f, g: F \rightarrow$ $F^{\prime}$ are $E_{\infty}$-maps with $\pi_{0}(f)=\pi_{0}(g)$. Then the functoriality of the Igusa tower implies that $K_{0}^{\vee}(f)=K_{0}^{\vee}(g)$. Hence $f$ and $g$ are homotopic as $E_{\infty^{-}}$ maps by the first part of the proposition.

Corollary 5.19. Let $G$ be a finite group of order prime to $p$. Suppose that $F$ and $F^{\prime}$ are $K(1)$-local, even-periodic, complex oriented $E_{\infty}$-ring spectra, that the rings $F_{0}$ and $F_{0}^{\prime}$ are $p$-torsion free, and that $F_{0}$ is the p-adic completion of a smooth $\mathbb{Z}_{p}$-algebra.

1. Any action of $G$ on $F$ in the homotopy category of $E_{\infty}$-ring spectra has a unique lift, up to weak equivalence, to an action of $G$ on $F$ in the category of $E_{\infty}$-ring spectra.

2. If $F$ and $F^{\prime}$ are equipped with actions of $G$ in the category of $E_{\infty}$-ring spectra, then the natural map

$$
\pi_{0} \operatorname{Map}_{E_{\infty}}^{G}\left(F, F^{\prime}\right) \rightarrow\left(H o m_{\psi-\theta-a l g}\left(K_{0}^{\vee} F, K_{0}^{\vee} F^{\prime}\right)\right)^{G},
$$

from the connected components of the derived mapping space of $G$ equivariant $E_{\infty}$-maps to $G$-equivariant maps of $\psi-\theta$-algebras, is an isomorphism. 
Proof. This combines Lemma 3.10 and the previous proposition.

We now state the main result allowing construction of $K(1)$-local objects and maps realizing algebraic data.

To help the reader navigate through the following statement, we remark that in the application $F$ will be the $K(1)$-localization of some $K(2)$-local $E_{\infty}$-ring spectrum and $T$ will be constructed as be a $K(1)$-local object together with a chromatic attaching map $T \rightarrow F$.

Theorem 5.20. Suppose $F$ is a $K(1)$-local, complex oriented $E_{\infty}$-ring spectrum such that $F_{*}$ is p-torsion free and concentrated in degrees congruent to 0 $\bmod (2 p-2)$. Let $T_{*}$ be a $p$-adic subring of $F_{*}$, with $T_{0}$ the $p$-adic completion of a smooth $\mathbb{Z}_{p}$-algebra. Suppose further that $T_{0} \subseteq F_{0}$ is closed under the operation $\theta$, that the orientation $M U_{*} \rightarrow F_{*}$ factors through $T_{*} \subseteq F_{*}$, and that the induced formal group law on $T_{*} /(p)$ is of strict height 1.

Then there exists a $K(1)$-local, complex oriented $E_{\infty}$-ring spectrum $T$, equipped with a map $T \rightarrow F$ of $E_{\infty}$-ring spectra, such that the induced map on homotopy groups is the given inclusion $T_{*} \rightarrow F_{*}$ and such that the orientation $M U \rightarrow F$ factors through an orientation $M U \rightarrow T$.

As a $K(1)$-local, complex orientable $E_{\infty}$-ring spectrum, $T$ is uniquely determined up to equivalence by $T_{*}$, the formal group over $T_{*}$ specified by the given map $M U_{*} \rightarrow T_{*}$, and the ring endomorphism of $T_{0}$ given by $x \mapsto x^{p}+p \theta(x)$. The map of $E_{\infty}$-ring spectra $T \rightarrow F$ is determined up to homotopy by its effect on homotopy groups mentioned above.

Proof. We will first construct $T(p)_{*}$ to be the first stage in the Igusa tower for $T_{*}$. Denote by $\mathbb{G}$ the formal group over $T_{*}$ classified by the given map $M U_{*} \rightarrow T_{*}$. Now, we define $T(p)_{*}$ to be the $T_{*}$-algebra which is the coordinate ring $\mathcal{O}_{\mathbb{G}^{\vee}[p] \backslash 0}$ parameterizing isomorphisms between the $p$-torsion $\mathbb{G}[p]$ of the formal group law on $T_{*}$ and the group of $p$-th roots of unity (see Lemma 5.11, item 2). This is a Galois extension of $T_{*}$ with Galois group $(\mathbb{Z} / p)^{\times}$. Naturality of the Igusa tower implies that the map $T_{*} \rightarrow F_{*}$ induces an equivariant isomorphism $F(p)_{*} \cong T(p)_{*} \otimes_{T_{*}} F_{*}$. The assumption that $F_{*}$ is concentrated in certain degrees implies that $F(p)_{0}=F_{0}$ and $T(p)_{0}=T_{0}$. 
We define graded rings

$$
\begin{aligned}
& A_{*}=\left(K_{*} \otimes_{M U P_{*}} M U P_{*} M U P \otimes_{M U P_{*}} T(p)_{*}\right)_{p}^{\wedge}, \text { and } \\
& B_{*}=\left(K_{*} \otimes_{M U P_{*}} M U P_{*} M U P \otimes_{M U P_{*}} F(p)_{*}\right)_{p}^{\wedge} .
\end{aligned}
$$

Both rings $A_{*}$ and $B_{*}$ are $p$-complete and even-periodic; this follows from the assumption on $F_{*}$. Both $A_{*}$ and $B_{*}$ have actions of $(\mathbb{Z} / p)^{\times}$through the rightmost factors in the tensor product. In addition, they carry actions of $\mathbb{Z}_{p}^{\times}$through Adams operations given by the coaction of the $p$-completed Hopf algebroid $\left(K_{*} \otimes_{M U P_{*}} M U P_{*} M U P \otimes_{M U P_{*}} K_{*}\right)_{p}^{\wedge}$. Specifically, for any $p$-adic unit $k \in \mathbb{Z}_{p}^{\times}$, the multiplication-by- $k$ map is a natural automorphism of any formal group law over a $p$-adic ring, and is classified on the universal example by a map

$$
[k]:\left(M U P_{*} M U P\right)_{p}^{\wedge} \rightarrow\left(M U P_{*}\right)_{p}^{\wedge} .
$$

Composing this map with the coalgebra action gives compatible actions of $\mathbb{Z}_{p}^{\times}$on $A_{*}$ and $B_{*}$.

By Lemma 5.4 there is an isomorphism, equivariant for $(\mathbb{Z} / p)^{\times}$and the action of the Adams operations, between $B_{*}$ and $K_{*}^{\vee} F(p)$.

As $T_{0}$ is closed under the operation $\theta$, the subring $A_{0} \subseteq B_{0}$ is also closed under the operation $\theta$, as follows. The ring $A_{0}$ is universal among $p$-adic $T(p)_{0}$-algebras $j: T(p)_{0} \rightarrow R$ equipped with an isomorphism $\widehat{\mathbb{G}}_{m} \rightarrow j^{*} \mathbb{G}$. Over the ring $B_{0}$, we have a composite isogeny

$$
\widehat{\mathbb{G}}_{m} \stackrel{\cong}{\rightarrow} \mathbb{G} \rightarrow \psi^{*} \mathbb{G}
$$

of degree $p$ by Theorem 2.1. As the formal multiplicative group has only one subgroup scheme of rank $p$, this factors uniquely as

$$
\widehat{\mathbb{G}}_{m} \stackrel{[p]}{\rightarrow} \widehat{\mathbb{G}}_{m} \cong \psi^{*} \mathbb{G}
$$

and the universal property then shows that the map $\psi: B_{0} \rightarrow B_{0}$ restricts to a map $A_{0} \rightarrow A_{0}$. The universal property also implies that this is a lift of Frobenius, and hence, since $A_{0}$ is $p$-torsion free, it has the structure of a $\psi$ - $\theta$-algebra. The action of $(\mathbb{Z} / p)^{\times}$commutes with this structure.

Lemma 5.5, item 1 implies that the maps $T(p)_{0} \rightarrow A_{0}$ and $F(p)_{0} \rightarrow B_{0}$ are ind-Galois extensions of $p$-adic rings with Galois group $\mathbb{Z}_{p}^{\times}$. We conclude that 
$A_{*}$ is the $p$-adic completion of an ind-étale extension of $T(p)_{*}$, that $T(p)_{0}$ is the $p$-adic completion of a smooth $\mathbb{Z}_{p}$-algebra, and that the continuous cohomology of $\mathbb{Z}_{p}^{\times}$with coefficients in $A_{*}$ vanishes. Applying Corollary 5.16 to $A_{*}$ implies that there exists a $K(1)$-local $E_{\infty}$-ring spectrum $T(p)$ equipped with an isomorphism $K_{*}^{\vee} T(p) \cong A_{*}$ as $\psi$ - $\theta$-algebras.

The homotopy of $T(p)$ is $T(p)_{*}$ by Theorem 5.1 , and is concentrated in even degrees. Therefore, $T(p)$ and $F(p)$ are even-periodic and complex orientable, their homotopy groups are $p$-torsion free, and $T(p)_{0}$ is the $p$-adic completion of a smooth $\mathbb{Z}_{p}$-algebra.

Proposition 5.18 implies that the inclusion $A_{*} \rightarrow B_{*}$ lifts to a unique map of $E_{\infty}$-ring spectra $f: T(p) \rightarrow F(p)$. As the inclusion is $(\mathbb{Z} / p)^{\times}$-equivariant, Corollary 5.19 implies that there exists, up to weak equivalence, a unique lift of this action of $(\mathbb{Z} / p)^{\times}$to $T(p)$, and a lift of $f$ to an equivariant map, where the action on the range is the action on $F(p)$ coming from the Igusa tower.

The description of the $K$-theory of $T(p)$, together with its Adams operations, determines the formal group of $T(p)$ as that coming from the given map $M U_{*} \rightarrow T(p)_{*}$. In fact, for any $K(1)$-local complex oriented multiplicative cohomology theory $F$, the action of the Adams operations on $K_{*}^{\vee} F$ precisely provides descent data for the formal group of multiplicative type carried by $F_{*}$. (One can instead view this in terms of the dual, étale group, which is locally isomorphic to $\mathbb{Q}_{p} / \mathbb{Z}_{p}$ and is classified by a character of the fundamental group with coefficients in $\mathbb{Z}_{p}^{\times}$.) Therefore, since this formal group law is lifted from one over $T_{*} \subseteq T(p)_{*}$, by Corollary 3.8 the orientation $M U \rightarrow F(p)$ lifts to an orientation $M U \rightarrow T(p)$.

Let $T$ be the homotopy-fixed point spectrum $T(p)^{h(\mathbb{Z} / p)^{\times}}$. We have a map $T \rightarrow F$ of $E_{\infty}$-ring spectra, and on homotopy groups this realizes the map of fixed subrings $T(p)_{*}^{(\mathbb{Z} / p)^{\times}} \rightarrow F(p)_{*}^{(\mathbb{Z} / p)^{\times}}$. By Lemma [5.11, item 3 this is precisely the map $T_{*} \rightarrow F_{*}$.

We then find that we have a composite splitting

$$
\operatorname{Map}_{E_{\infty}}(T, F) \rightarrow \operatorname{Map}_{E_{\infty}}^{(\mathbb{Z} / p)^{\times}}(T(p), F(p)) \rightarrow \operatorname{Map}_{E_{\infty}}(T, F)
$$

given by first taking the first stage in the Igusa tower, followed by taking homotopy fixed points. Upon applying $\pi_{0}$, homotopical uniqueness of the equivariant lift $T(p) \rightarrow F(p)$ then implies uniqueness of the map $T \rightarrow F$. 
Corollary 5.13 implies that the orientation $M U \rightarrow T(p)$ has a unique lift $M U \rightarrow T$.

\section{$6 \quad K(1)$-local realization}

In this section, we will begin with a realization problem $(A, \mathbb{G})$ as in Definition 3.1, together with the unique solution $R_{K(2)}$ to the realization problem for $A_{K(2)}$ as in Theorem 4.5. We will extend this to study solutions to the realization problem for $A_{p}^{\wedge}$, using the $K(1)$-local $E_{\infty}$-ring spectrum $R_{K(2), K(1)}=L_{K(1)} R_{K(2)}$.

\section{1 $K(1)$-localized algebras}

The following result will verify the assumptions of Theorem 5.20 and will lead to the construction of the chromatic attaching map $R_{K(1)} \rightarrow R_{K(2), K(1)}$ in Section 6.2.

Proposition 6.1. Suppose that $(A, \mathbb{G})$ is a realization problem, and $R_{K(2)}$ is the solution to the realization problem for $A_{K(2)}$ given by Theorem 4.5. Let $F$ be the $K(1)$-localization $R_{K(2), K(1)}=L_{K(1)} R_{K(2)}$, and let $B=A_{K(1)}$. For brevity, we define $x$ to be $v_{1}^{p+1} v_{2}^{-1}$.

- The spectrum $F$ is a $K(1)$-local complex oriented $E_{\infty}$-ring spectrum, with $F_{*}$ concentrated in even degrees and $p$-torsion free.

- The ring $B$ maps identically into a p-adic subring of $F_{*}$, with $B_{0} \cong$ $\mathbb{Z}\left[x^{-1}\right]_{p}^{\wedge}$ the $p$-adic completion of a smooth $\mathbb{Z}_{p}$-algebra.

- The orientation $M U_{*} \rightarrow F_{*}$ factors through $B \subseteq F_{*}$.

Proof. We consider $F$ complex oriented by the composite

$$
M U \rightarrow R_{K(2)} \rightarrow R_{K(2), K(1)}=F,
$$

where the first map is the orientation which is part of the solution to our realization problem for $A_{K(2)}$ and the second map is the canonical localization map. 
We first compute the homotopy of the $K(1)$-localization $F$. Application of [Hov97, Corollary 1.5.5] allows us to conclude that the $K(1)$-localization is $F=\left(v_{1}^{-1} R_{K(2)}\right)_{p}^{\wedge}$. We then have the following (see Remarks 3.5 and 3.6):

$$
F_{*} \cong\left(A_{K(2)}\right)_{K(1)} \cong\left(\mathbb{Z}_{p}((x))_{p}^{\wedge}\right)\left[v_{1}^{ \pm 1}\right]
$$

From this we find that the map

$$
B=A_{K(1)}=\mathbb{Z}_{p}\left[x^{-1}\right]_{p}^{\wedge}\left[v_{1}^{ \pm 1}\right] \rightarrow F_{*}
$$

is injective, and the orientation factors as $M U_{*} \rightarrow A \hookrightarrow B \hookrightarrow F_{*}$, where the first map is part of our realization problem.

\subsection{Application of obstruction theory}

Theorem 6.2. Suppose that $(A, \mathbb{G})$ is a realization problem, and that $R_{K(2)}$ is the solution to the lifting problem for $A_{K(2)}$ given by Theorem 4.5. Let $F$ be the $K(1)$-localization $R_{K(2), K(1)}$, and let $B \subseteq F_{*}$ be the subring which is the image of $A_{K(1)}$ as in Proposition 6.1.

Then $B_{0}$ is closed under the power operation $\theta$, defined on $F_{0}$ as in section 5.4. if and only if there exists a $K(1)$-local $E_{\infty}$-ring spectrum $T$ solving the realization problem for $B$ together with a "chromatic attaching map" of $E_{\infty}$ ring spectra $\alpha^{\text {chrom }}: T \rightarrow F$, such that the induced map on homotopy groups is isomorphic to the inclusion $B \rightarrow F_{*}$. Any two such spectra $T$ are equivalent as $E_{\infty}$-ring spectra over $F$.

Under these circumstances, the orientation $M U \rightarrow F$ factors as $M U \rightarrow T \rightarrow$ $F$.

Proof. The necessity follows from the naturality of the operation $\theta$. To show sufficiency, Proposition 6.1, together with the assumptions on the power operation $\theta$, show that the subring $B \subseteq F_{*}$ satisfies the assumptions of Theorem 5.20 .

Definition 6.3. Suppose that $(A, \mathbb{G})$ is a realization problem such that the image of $\left(A_{K(1)}\right)_{0}$ in $\left(A_{K(2), K(1)}\right)_{0}$ is closed under the power operation $\theta$. 
Then let $R_{K(1) \vee K(2)}$ be the $E_{\infty}$-ring spectrum formed as the homotopy pullback in the following diagram:

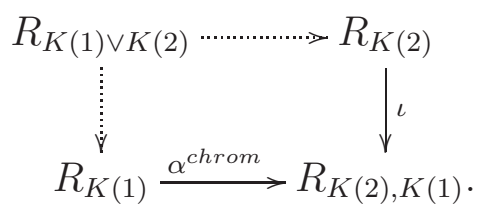

Here, $R_{K(2)}$ is as in Theorem 4.5, $\iota$ is the canonical localization map, and $\alpha^{\text {chrom }}$ is the chromatic attaching map from Theorem 6.2.

Proposition 6.4. Under the conditions of Definition [6.3, the connective cover of $R_{K(1) \vee K(2)}$ solves the realization problem for $A_{p}^{\wedge} \cong \mathbb{Z}_{p}\left[v_{1}, v_{2}\right]$, and any two such solutions are equivalent.

Proof. The forgetful functor from $E_{\infty}$-ring spectra to spectra preserves homotopy limits. The homotopy groups of all spectra involved in the solid part of (6.2) are concentrated in even degrees, and $\pi_{*}(\iota)$ and $\pi_{*}\left(\alpha^{\text {chrom }}\right)$ are injective. Therefore, for all $n \in \mathbb{Z}$ we have

$$
\begin{gathered}
\pi_{2 n} R_{K(1) \vee K(2)}=\pi_{2 n} R_{K(2)} \cap \pi_{2 n} R_{K(1)} \text { and } \\
\pi_{2 n-1} R_{K(1) \vee K(2)}=\operatorname{coker}\left(\pi_{2 n} R_{K(2)} \oplus \pi_{2 n} R_{K(1)} \rightarrow \pi_{2 n} R_{K(2), K(1)}\right) .
\end{gathered}
$$

Explicitly, we have the following.

$$
\begin{aligned}
\pi_{2 n} R_{K(2)}=\left\{\sum_{k+(p+1) l=n, k \geq 0} c_{k, l} v_{1}^{k} v_{2}^{l} \mid c_{k, l} \in \mathbb{Z}_{p}\right\} & \left\{\sum_{0 \leq k \equiv n(p+1)} c_{k} v_{1}^{k} v_{2}^{\frac{n-k}{p+1}} \mid c_{k} \in \mathbb{Z}_{p}\right\} \\
= & \left\{\sum_{\pi_{2 n} R_{K(1)}=} \sum_{k+(p+1) l=n, l \geq 0} c_{k, l} v_{1}^{k} v_{2}^{l} \mid c_{k, l} \in \mathbb{Z}_{p}, c_{k, l} \rightarrow 0 \text { as } k \rightarrow-\infty\right\} \\
= & \left\{\sum_{0 \leq l} c_{l} v_{1}^{n-(p+1) l} v_{2}^{l} \mid c_{l} \in \mathbb{Z}_{p}, c_{l} \rightarrow 0\right\}
\end{aligned}
$$




$$
\begin{aligned}
\pi_{2 n} R_{K(2), K(1)} & =\left\{\sum_{k+(p+1) l=n} c_{k, l} v_{1}^{k} v_{2}^{l} \mid c_{k, l} \in \mathbb{Z}_{p}, c_{k, l} \rightarrow 0 \text { as } k \rightarrow-\infty\right\} \\
& =\left\{\sum_{k \in n+(p+1) \mathbb{Z}} c_{k} v_{1}^{k} v_{2}^{\frac{n-k}{p+1}} \mid c_{k} \in \mathbb{Z}_{p}, c_{k} \rightarrow 0 \text { for } k \rightarrow-\infty\right\} .
\end{aligned}
$$

If $n \geq 0$, every element in $\pi_{2 n} R_{K(2), K(1)}$ is a sum of elements in the images of the other two groups. Therefore, for all $n \geq 0$ we have $\pi_{2 n-1} R_{K(1) \vee K(2)}=0$ and

$$
\pi_{2 n} R_{K(1) \vee K(2)}=\left\{\sum_{k+(p+1) l=n, k \geq 0, l \geq 0} c_{k, l} v_{1}^{k} v_{2}^{l} \mid c_{k, l} \in \mathbb{Z}_{p}\right\},
$$

which is the portion of $A_{p}^{\wedge} \cong \mathbb{Z}_{p}\left[v_{1}, v_{2}\right]$ concentrated in degree $2 n$. Hence the connective cover $R_{K(1) \vee K(2)}[0, \infty)$, which we denote by $R_{p}^{\wedge}$, is an $E_{\infty}$-ring spectrum with homotopy $\pi_{*} R_{p}^{\wedge} \simeq A_{p}^{\wedge}$. Since this is concentrated in even dimensions, $R_{p}^{\wedge}$ is complex orientable and it remains to see that the resulting formal group is the one given in the realization problem.

As $M U$ is connective, orientations of $R_{p}^{\wedge}$ are in bijective correspondence with orientations of $R_{K(1) \vee K(2)}$. Moreover, we know that the formal group laws of $R_{K(1)}$ and $R_{K(2)}$ are the formal group laws pushed forward from A. Applying Lemma 3.9, we find that $R_{p}^{\wedge}$ is complex orientable with formal group law pushed forward from the map $A \rightarrow \pi_{*} R_{p}^{\wedge}$.

This shows that $R_{p}^{\wedge}$ is a solution to the realization problem for $A_{p}^{\wedge}$, and we now address uniqueness.

Given any other solution $R^{\prime}$, Theorem 4.5 shows that there exists an equivalence of realizations $R_{K(2)} \rightarrow R_{K(2)}^{\prime}$, and Theorem 6.2 shows that there exists an equivalence $R_{K(1)} \rightarrow R_{K(1)}^{\prime}$ of realizations compatible with the chromatic attaching maps. Taking the connective cover of the induced equivalence $R_{K(1) \vee K(2)} \rightarrow R_{K(1) \vee K(2)}^{\prime}$ gives the desired result. 


\section{$7 \quad K(0)$-local realization}

Theorem 7.1. Suppose that $(A, \mathbb{G})$ is a realization problem. Then $(A, \mathbb{G})$ has a solution if and only if the degree-zero portion $\left(A_{K(1)}\right)_{0} \subseteq\left(A_{K(2), K(1)}\right)_{0}$ is stable under the power operation $\theta$. If such a solution exists, it is unique up to equivalence.

Proof. It is clear that closure under $\theta$ is a necessary condition, so let us assume it holds and establish that our realization problem has a solution, unique up to equivalence. Let $R_{\mathbb{Z}_{p}}=R_{p}^{\wedge}$ be the solution to the realization problem for $A_{p}^{\wedge}$ from Proposition 6.4, and define $R_{\mathbb{Q}_{p}}$ to be the rationalization of $R_{\mathbb{Z}_{p}}$. Let $R_{\mathbb{Q}}$ be the free $E_{\infty}-\mathbb{H} \mathbb{Q}$-algebra on $S^{2 p-2} \vee S^{2 p^{2}-2}$. Then $\pi_{*} R_{\mathbb{Q}}$ is isomorphic to $A_{K(0)} \cong \mathbb{Q}\left[v_{1}, v_{2}\right]$, and $R_{\mathbb{Q}}$ is a solution to the realization problem for $A_{K(0)}$.

Proposition 6.4 and rational homotopy theory imply that there exists an arithmetic attaching map of $E_{\infty}-\mathbb{H} \mathbb{Q}$-algebras

$$
\alpha^{\text {arith }}: R_{\mathbb{Q}} \rightarrow R_{\mathbb{Q}_{p}}
$$

inducing the inclusion $\mathbb{Q}\left[v_{1}, v_{2}\right] \rightarrow \mathbb{Q}_{p}\left[v_{1}, v_{2}\right]$ on homotopy groups.

Let $R$ be the $E_{\infty}$-ring spectrum formed as the homotopy pullback in the following diagram:

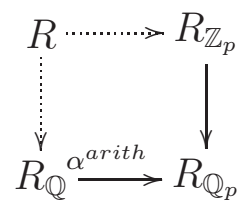

In a similar manner to the proof of Proposition 6.4, we find that the pullback diagram defining $R$ gives rise to the following Mayer-Vietoris square on homotopy groups:

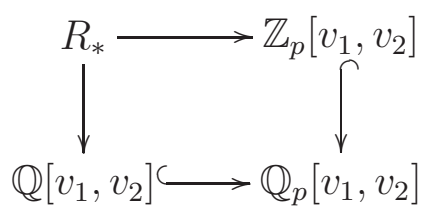

Observe that, since $\mathbb{Q}_{p}=\mathbb{Q}+\mathbb{Z}_{p}$, we have $\pi_{2 n-1} R=0$ for all $n \geq 0$. The above diagram is then a pullback diagram, and the homotopy groups of $R$ 
map isomorphically to the subring which is the image of $A=\mathbb{Z}_{(p)}\left[v_{1}, v_{2}\right]$. The formal group laws of $R_{\mathbb{Z}_{p}}$ and $R_{\mathbb{Q}}$ are the formal group laws pushed forward from $A$. Applying Lemma [3.9, we find that $R$ is complex orientable with formal group law pushed forward from the isomorphism $A \rightarrow R_{*}$. Therefore, $R$ is a solution to the realization problem for $A$ itself.

Given any other solution $T$ to the realization problem, Proposition 6.4 implies that there exists an equivalence $R_{\mathbb{Z}_{p}} \rightarrow T_{p}^{\wedge}$ of realizations for $A_{p}^{\wedge}$. As $R_{\mathbb{Q}}$ is free, in order to construct a map of $E_{\infty}-\mathbb{H} \mathbb{Q}$-algebras $R_{\mathbb{Q}} \rightarrow T_{\mathbb{Q}}$ compatible with the arithmetic attaching maps it suffices for the images of the generators $v_{1}, v_{2}$ in $\pi_{*} T_{\mathbb{Q}_{p}}$ to lift to $\pi_{*} T_{\mathbb{Q}}$. However, this follows because an equivalence of realizations preserves the subring of $A_{p}^{\wedge}$ which is the image of $A$.

\section{An algebraic description of $\theta$ and the proof of Theorem 1.1}

In Section 8.1 we will give an algebraic characterization of the power operation $\theta$ figuring in Theorem 7.1. Section 8.2 uses this, and the moduli of generalized elliptic curves with $\Gamma_{1}(3)$-level structure, to produce a realization problem at the prime 2 meeting the assumptions of Theorem 7.1. This will complete the proof of Theorem 1.1 .

\subsection{Computing $\theta$}

Let $(A, \mathbb{G})$ be a (generalized $\mathrm{BP}\langle 2\rangle$-)realization problem. Crucially using the Goerss-Hopkins-Miller theorem, we obtained an operation $\theta$ on $\left(A_{K(2), K(1)}\right)_{0}$, which is a mixed-characteristic discrete valuation ring. Since this ring is torsion-free, $\theta$ is determined by the ring endomorphism

$$
x \mapsto\left(\psi^{p}\right)^{t o p}(x):=x^{p}+p \cdot \theta(x)
$$

of $\left(A_{K(2), K(1)}\right)_{0}$. This map $\left(\psi^{p}\right)^{t o p}$ is a lift of Frobenius: a ring endomorphism with $\left(\psi^{p}\right)^{\text {top }}(x) \equiv x^{p} \bmod p$. In this section we will explain how to define $\left(\psi^{p}\right)^{t o p}$ without homotopy theory, and in particular without solving the realization problem for $A_{K(2)}$. This is essentially a consequence of work of 
Ando-Hopkins-Strickland relating the power operations on Lubin-Tate spectra to their naturally defined descent data for level structures AHS04, Section 12.4].

Recall the associated Lubin-Tate ring $B \supseteq A_{K(2)}$ from Definition 4.1, a $G$ Galois extension for the group $G=\mathbb{F}_{p^{2}}^{\times} \rtimes \operatorname{Gal}\left(\mathbb{F}_{p^{2}} / \mathbb{F}_{p}\right)$. Form the pushout of rings

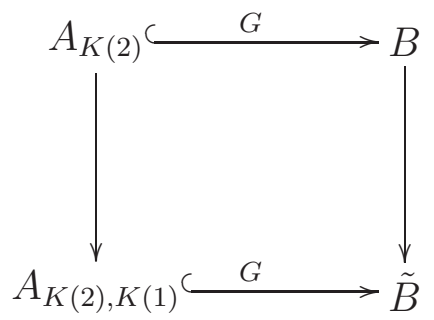

along the canonical ring homomorphism $A_{K(2)} \rightarrow A_{K(2), K(1)}$. The base change $\mathbb{G}_{B}$ over $B$ of the given formal group $\mathbb{G}$ is a Lubin-Tate formal group by Remark 4.3, and from Remark 3.5 we see that $\tilde{B} \cong\left(v_{1}^{-1} B\right)_{p}^{\wedge}$. This shows that $\mathbb{G}_{B}$ is a $p$-divisible group of height 2 which is a universal deformation of its special fiber. The base change $\mathbb{G}_{\tilde{B}}$ of the $p$-divisible group $\mathbb{G}_{B}$ to $\tilde{B}$ is ordinary [HT01, Lemma II.1.1, 4], so there is an extension of $p$-divisible groups over $\tilde{B}$

$$
0 \rightarrow \mathbb{G}_{\tilde{B}}^{f o r} \stackrel{\iota}{\rightarrow} \mathbb{G}_{\tilde{B}} \rightarrow \mathbb{G}_{\tilde{B}}^{e t} \rightarrow 0
$$

with $\mathbb{G}_{\tilde{B}}^{\text {for }}\left(\right.$ resp. $\left.\mathbb{G}_{\tilde{B}}^{e t}\right)$ a formal (resp. étale) p-divisible group of height 1 . The kernel $\mathbb{G}_{\tilde{B}}^{\text {for }}[p] \subseteq \mathbb{G}_{\tilde{B}}^{\text {for }}$ of multiplication by $p$ is the unique subgroup of $\mathbb{G}_{\tilde{B}}^{\text {for }}$ of order $p$. We obtain a subgroup $C$ of order $p$ as the image $\iota\left(\mathbb{G}_{\tilde{B}}^{f o r}[p]\right) \subseteq \mathbb{G}_{\tilde{B}}$, called the canonical subgroup. By construction, the mod- $p$ reduction of the quotient of $\mathbb{G}_{\tilde{B}}$ by its canonical subgroup has canonical isomorphisms

$$
{\overline{\mathbb{G}_{\tilde{B}}} / C}_{\overline{\mathbb{G}}_{\tilde{B}}}(p) \cong \overline{\mathbb{G}} \tilde{B} .
$$

(As in Section $2.4, \overline{\mathbb{G}} \tilde{B}^{(p)}$ denotes the pullback along the Frobenius isogeny.) The latter isomorphism occurs because $\overline{\mathbb{G}_{\tilde{B}}}$ is defined over $\mathbb{F}_{p}$. We conclude from [Lau10, Proposition 7.1] that there is a unique lift of Frobenius $\psi: \tilde{B} \rightarrow$ $\tilde{B}$ such that there is an isomorphism of $p$-divisible groups over $\tilde{B}$

$$
\psi^{*} \mathbb{G}_{\tilde{B}} \cong \mathbb{G}_{\tilde{B}} / C .
$$

Since $\mathbb{G}_{\tilde{B}}$ comes by base change from $A_{K(2), K(1)} \subseteq \tilde{B}, \psi$ restricts to a lift of Frobenius $\left(\psi^{p}\right)^{\text {alg }}$ on $A_{K(2), K(1)}$. The construction of $\left(\psi^{p}\right)^{\text {alg }}$ involves no homotopy theory, so the following result achieves the goal of this section. 
Proposition 8.1. In the above situation we have $\left(\psi^{p}\right)^{\text {top }}=\left(\psi^{p}\right)^{\text {alg }}$.

Proof. It is enough to see that $\psi$ is equal to $\left(\psi^{p}\right)^{t o p} \otimes \mathrm{id}_{\tilde{B}}$. This is true because, by equation (2.2) in Section 2.4, the topologically constructed lift of Frobenius $\left(\psi^{p}\right)^{t o p} \otimes \mathrm{id}_{\tilde{B}}$ satisfies property (8.1), which characterizes the algebraically constructed lift $\psi$.

Remark 8.2. It is indispensable to use non-formal $p$-divisible groups to obtain an algebraic characterization of $\left(\psi^{p}\right)^{t o p}$ as above. The base-change of the formal group $\mathbb{G}_{B}$ to $\tilde{B}$ is $\mathbb{G}_{\tilde{B}}^{\text {for }}$, and the first results in Section 2.4 imply that one cannot recover $\left(\psi^{p}\right)^{\text {alg }}$ from knowledge of $\mathbb{G}_{\tilde{B}}^{\text {for }} \subseteq \mathbb{G}_{\tilde{B}}$ alone.

\subsection{Proof of Theorem 1.1}

In this section we complete the proof of Theorem 1.1 by constructing a realization problem $(A, \mathbb{G})$ at the prime 2 which meets the assumptions of Theorem 7.1 .

Define $A$ to be the graded ring $\mathbb{Z}_{(2)}[a, b]$ with grading $|a|=2,|b|=6$. Consider the following Weierstrass equation over $A$ :

$$
y^{2}+a \cdot x y+b \cdot y=x^{3} .
$$

We remark this is a universal generalized elliptic curve with a $\Gamma_{1}(3)$-level structure; this connection will be exploited in [LN].

Equation (8.2) determines a graded formal group law $\mathbb{G}$ over $A$ with respect to the coordinate $-x / y$.

Proposition 8.3. This pair $(A, \mathbb{G})$ is a realization problem at the prime 2 .

Proof. From [Lau04, Lemma 1], the 2-primary Hazewinkel generators $v_{1}$ and $v_{2}$ are mapped to $a$ and $b$ respectively.

Resuming notation from Section 8.1, our remaining task is to compute $\theta$ (or equivalently $\left.\left(\psi^{2}\right)^{a l g}\right)$ for the present realization problem. We observe that by 
defining $t$ to be $a u^{-1}$, where $a$ is the image of $a \in A$ and $u \in B_{2}$ satisfies $u^{3}=b$, we have

$$
B_{0}=\mathbb{W}\left(\mathbb{F}_{4}\right) \llbracket t \rrbracket .
$$

Over $B_{0}$, the Weierstrass curve of Equation (8.2) becomes isomorphic to the following curve:

$$
E: y^{2}+t \cdot x y+y=x^{3}
$$

The key computational result, which at present lacks an analogue at primes other than 2 , is the following.

Proposition 8.4 ([Rezb, Section 3]). The universal isogeny of degree 2 with domain $E$ is defined over $B_{0}[d] /\left(d^{3}-t d-2\right)$, and has range the elliptic curve

$$
y^{2}+\left(t^{2}+3 d-t d^{2}\right) x y+y=x^{3} .
$$

The kernel of this isogeny is generated by the 2-torsion point with coordinates $(x / y, 1 / y)=\left(-d, d^{3}\right)$.

This first implies that there is a unique $\alpha \in \tilde{B}=\left(t^{-1} B_{0}\right)_{2}^{\wedge}$ satisfying $\alpha^{3}-$ $t \alpha-2=0$, because solutions of this cubic equation define points of order 2 on the curve. There is a unique such point $P \in E(\tilde{B})$ defined over the complete discrete valuation ring $\tilde{B}$ because the curve is ordinary there. (The existence of a unique $\alpha$ can also be checked directly using Hensel's lemma.) Second, since $\left(\left(\psi^{2}\right)^{a l g}\right)^{*} E \cong E /\{1, P\}$, Rezk's computations imply that we have

$$
\left(\psi^{2}\right)^{a l g}(t)=t^{2}+3 \alpha-\alpha^{2} t=t^{-1}\left(t^{3}+3 \alpha t-(\alpha t)^{2}\right) .
$$

The element $\alpha \in \tilde{B}$ is divisible by two. Defining $c$ to be $\frac{1}{2} \alpha t \in \tilde{B}$, the above cubic equation $\alpha^{3}-t \alpha-2=0$ for $\alpha$ is equivalent to the equation

$$
c=-1+4 t^{-3} c^{3} \text {. }
$$

This can recursively be solved to yield

$$
c=-1-4 t^{-3}-48 \cdot t^{-6}-192 \cdot t^{-9} \cdots \in \mathbb{Z}_{2}\left[t^{-3}\right]_{2}^{\wedge} .
$$

With our present choice of coordinate, the inclusion $\left(A_{K(1)}\right)_{0} \subseteq\left(A_{K(2), K(1)}\right)_{0}$ is the inclusion of the subring $\mathbb{Z}_{2}\left[t^{-3}\right]_{2}^{\wedge} \subseteq \mathbb{Z}_{2}\left(\left(t^{3}\right)\right)_{2}^{\wedge}$. Combining the above, one verifies that $\left(\psi^{2}\right)^{\text {alg }}\left(t^{-3}\right) \in \mathbb{Z}_{2}\left[t^{-3}\right]_{2}^{\wedge}$. In combination with Proposition 8.1 this establishes the following.

Proposition 8.5. The subring $\left(A_{K(1)}\right)_{0} \subseteq\left(A_{K(2), K(1)}\right)_{0}$ is stable under $\theta$.

In combination with Theorem [7.1, this proves Theorem 1.1, 


\section{References}

[AHS04] Matthew Ando, Michael J. Hopkins, and Neil P. Strickland, The sigma orientation is an $H_{\infty}$ map, Amer. J. Math. 126 (2004), no. 2, 247-334. MR 2045503 (2005d:55009)

[And95] Matthew Ando, Isogenies of formal group laws and power operations in the cohomology theories $E_{n}$, Duke Math. J. 79 (1995), no. 2, 423-485. MR 1344767 (97a:55006)

[AR02] Christian Ausoni and John Rognes, Algebraic K-theory of topological K-theory, Acta Math. 188 (2002), no. 1, 1-39. MR 1947457 (2004f:19007)

[Bak00] Andrew Baker, $I_{n}$-local Johnson-Wilson spectra and their Hopf algebroids, Doc. Math. 5 (2000), 351-364 (electronic).

[Bak01] On the Adams $E_{2}$-term for elliptic cohomology, Homotopy methods in algebraic topology (Boulder, CO, 1999), Contemp. Math., vol. 271, Amer. Math. Soc., Providence, RI, 2001, pp. 1-15. MR 1831344 (2002c:55012)

[Beh] Mark Behrens, Notes on the construction of $\operatorname{tmf}$, available at: http://www-math.mit.edu/ mbehrens/papers/buildTMF.pdf.

[BL10] Mark Behrens and Tyler Lawson, Topological automorphic forms, Mem. Amer. Math. Soc. 204 (2010), no. 958, xxiv+141. MR 2640996

[BM08] Andrew J. Blumberg and Michael A. Mandell, The localization sequence for the algebraic $K$-theory of topological $K$-theory, Acta Math. 200 (2008), no. 2, 155-179. MR 2413133 (2009f:19003)

[BMMS86] Robert R. Bruner, J. Peter May, James E. McClure, and Mark Steinberger, $H_{\infty}$ ring spectra and their applications, Lecture Notes in Mathematics, vol. 1176, Springer-Verlag, Berlin, 1986. MR 836132 (88e:55001)

[BR07] Andrew Baker and Birgit Richter, Realizability of algebraic Galois extensions by strictly commutative ring spectra, Trans. Amer. 
Math. Soc. 359 (2007), no. 2, 827-857 (electronic). MR 2255198 (2007m:55007)

[Coo78] George Cooke, Replacing homotopy actions by topological actions, Trans. Amer. Math. Soc. 237 (1978), 391-406. MR 0461544 (57 \#1529)

[Dav06] Daniel G. Davis, Homotopy fixed points for $L_{K(n)}\left(E_{n} \wedge X\right)$ using the continuous action, J. Pure Appl. Algebra 206 (2006), no. 3, 322-354. MR 2235364 (2007b:55008)

[DH04] Ethan S. Devinatz and Michael J. Hopkins, Homotopy fixed point spectra for closed subgroups of the Morava stabilizer groups, Topology 43 (2004), no. 1, 1-47. MR 2030586 (2004i:55012)

[DT] Daniel G. Davis and Takeshi Torii, Every $K(n)$-local spectrum is the homotopy fixed points of its Morava module, to appear in Proc. Amer. Math. Soc.

[EKMM97] Anthony D. Elmendorf, Igor Kriz, Michael A. Mandell, and J. Peter May, Rings, modules, and algebras in stable homotopy theory, Mathematical Surveys and Monographs, vol. 47, American Mathematical Society, Providence, RI, 1997, With an appendix by M. Cole. MR 1417719 (97h:55006)

[GH] Paul G. Goerss and Michael J. Hopkins, Moduli problems for structured ring spectra, available at: http://www.math.northwestern.edu/ pgoerss/spectra/obstruct.pdf.

[GH04] _ Moduli spaces of commutative ring spectra, Structured ring spectra, London Math. Soc. Lecture Note Ser., vol. 315, Cambridge Univ. Press, Cambridge, 2004, pp. 151-200. MR 2125040 (2006b:55010)

[GM95] John P. C. Greenlees and J. Peter May, Completions in algebra and topology, Handbook of algebraic topology, North-Holland, Amsterdam, 1995, pp. 255-276. MR 1361892 (96j:55011)

[Goe] Paul G. Goerss, Quasi-coherent sheaves on the moduli stack of formal groups, available at: http://www.math.northwestern.edu/ ${ }^{\sim}$ pgoerss/papers/modfg.pdf. 
[Goe04] (Pre-)sheaves of ring spectra over the moduli stack of formal group laws, Axiomatic, enriched and motivic homotopy theory, NATO Sci. Ser. II Math. Phys. Chem., vol. 131, Kluwer Acad. Publ., Dordrecht, 2004, pp. 101-131. MR 2061853 (2005d:55007)

[Goe09]_, Realizing families of Landweber exact homology theories, New topological contexts for Galois theory and algebraic geometry (BIRS 2008), Geom. Topol. Monogr., vol. 16, Geom. Topol. Publ., Coventry, 2009, pp. 49-78. MR 2544386 (2010h:55007)

[Goe10]_, Topological modular forms [after Hopkins, Miller and Lurie], Astérisque (2010), no. 332, Exp. No. 1005, viii, 221-255, Séminaire Bourbaki. Volume 2008/2009. Exposés 997-1011. MR 2648680

[HL10] Michael Hill and Tyler Lawson, Automorphic forms and cohomology theories on Shimura curves of small discriminant, Adv. Math. 225 (2010), no. 2, 1013-1045. MR 2671186

[Hop] Michael J. Hopkins, $\quad K(1)$-local $E_{\infty^{-}}$ ring spectra, preprint, available at:http://www.math.rochester.edu/people/faculty/doug/otherpapers/knlocal.pdf.

[Hov] Mark Hovey, Some spectral sequences in Morava E-theory, available at: http://math.wesleyan.edu/ ${ }^{\sim}$ mhovey/papers/morava-ESS.pdf.

[Hov95]_— Bousfield localization functors and Hopkins' chromatic splitting conjecture, The Čech centennial (Boston, MA, 1993), Contemp. Math., vol. 181, Amer. Math. Soc., Providence, RI, 1995, pp. 225-250. MR 1320994 (96m:55010)

[Hov97],$v_{n}$-elements in ring spectra and applications to bordism theory, Duke Math. J. 88 (1997), no. 2, 327-356. MR 1455523 (98d:55017)

[Hov08] - Morava E-theory of filtered colimits, Trans. Amer. Math. Soc. 360 (2008), no. 1, 369-382 (electronic). MR 2342007 (2008g:55007) 
[HS99] Mark Hovey and Neil Strickland, Morava K-theories and localisation, Mem. Amer. Math. Soc. 139 (1999), no. 666, viii+100. MR 1601906 (99b:55017)

[HT01] Michael Harris and Richard Taylor, The geometry and cohomology of some simple Shimura varieties, Annals of Mathematics Studies, vol. 151, Princeton University Press, Princeton, NJ, 2001, With an appendix by Vladimir G. Berkovich. MR 1876802 (2002m:11050)

[JW73] David C. Johnson and W. Stephen Wilson, Projective dimension and Brown-Peterson homology, Topology 12 (1973), 327-353. MR 0334257 (48 \#12576)

[Lau03] Gerd Laures, An $E_{\infty}$ splitting of spin bordism, Amer. J. Math. 125 (2003), no. 5, 977-1027. MR 2004426 (2004g:55007)

[Lau04] _ $K(1)$-local topological modular forms, Invent. Math. 157 (2004), no. 2, 371-403. MR 2076927 (2005h:55003)

[Lau10] Eike Lau, Tate modules of universal p-divisible groups, Compos. Math. 146 (2010), no. 1, 220-232. MR 2581248 (2011d:14082)

[Law10] Tyler Lawson, Structured ring spectra and displays, Geom. Topol. 14 (2010), no. 2, 1111-1127. MR 2629902

[Laz03] Andrey Lazarev, Towers of $M U$-algebras and the generalized Hopkins-Miller theorem, Proc. London Math. Soc. (3) 87 (2003), no. 2, 498-522. MR 1990937 (2004c:55006)

[LN] Tyler Lawson and Niko Naumann, Realization of modules over the steenrod algebra and a multiplicative tmf-orientation at 2, in preperation.

[LT66] Jonathan Lubin and John Tate, Formal moduli for oneparameter formal Lie groups, Bull. Soc. Math. France 94 (1966), 49-59. MR 0238854 (39 \#214)

[Lur09] Jacob Lurie, A survey of elliptic cohomology, Algebraic topology, Abel Symp., vol. 4, Springer, Berlin, 2009, pp. 219-277. MR 2597740 
[Mil80] James S. Milne, Étale cohomology, Princeton Mathematical Series, vol. 33, Princeton University Press, Princeton, N.J., 1980. MR 559531 (81j:14002)

[MS93] James E. McClure and Ross E. Staffeldt, On the topological Hochschild homology of bu. I, Amer. J. Math. 115 (1993), no. 1, 1-45. MR 1209233 (94d:55020)

[Reza] Charles Rezk, Lectures on power operations, available at: http://www.math.uiuc.edu/〜rezk/papers.html.

[Rezb] _ Power operations for Morava E-theory of height 2 at the prime 2, arXiv:0812.1320.

[Rez98] _ Notes on the Hopkins-Miller theorem, Homotopy theory via algebraic geometry and group representations (Evanston, IL, 1997), Contemp. Math., vol. 220, Amer. Math. Soc., Providence, RI, 1998, pp. 313-366. MR 1642902 (2000i:55023)

[Rog08] John Rognes, Galois extensions of structured ring spectra. Stably dualizable groups, Mem. Amer. Math. Soc. 192 (2008), no. 898, viii+137. MR 2387923 (2009c:55007)

[Str99a] Neil P. Strickland, Formal schemes and formal groups, Homotopy invariant algebraic structures (Baltimore, MD, 1998), Contemp. Math., vol. 239, Amer. Math. Soc., Providence, RI, 1999, pp. 263-352. MR 1718087 (2000j:55011)

[Str99b] _ Products on MU-modules, Trans. Amer. Math. Soc. 351 (1999), no. 7, 2569-2606.

[Szy11] Markus Szymik, Crystals and derived local moduli for ordinary K3 surfaces, Adv. Math. 228 (2011), no. 1, 1-21.

[Tat67] John Tate, $p$ - divisible groups., Proc. Conf. Local Fields (Driebergen, 1966), Springer, Berlin, 1967, pp. 158-183. MR 0231827 (38 \#155)

[Tat76] Relations between $K_{2}$ and Galois cohomology, Invent. Math. 36 (1976), 257-274. MR 0429837 (55 \#2847) 
[TO70] John Tate and Frans Oort, Group schemes of prime order, Ann. Sci. École Norm. Sup. (4) 3 (1970), 1-21. MR 0265368 (42 \#278) 